\title{
Validation tests of the full-discontinuous Galerkin / extrinsic cohesive law framework of Kirchhoff-Love shells
}

\author{
Gauthier Becker · Ludovic Noels
}

Received: date / Accepted: date

\begin{abstract}
Due to its ability to account for discontinuities, the discontinuous Galerkin (DG) method presents two main advantages for modeling crack initiations and propagation. On the one hand, it provides an easy way to insert the cohesive elements during the simulation and therefore avoids the drawbacks inherent to the use of an extrinsic cohesive law. On the other hand, the capture of complex crack path requires very thin meshes and the recourse to a parallel implementation of DG formulations exhibits a high scalability of the resolution scheme.

Recently, the authors developed such a DG-fracture framework for Kirchhoff-Love shells in the linear and non-linear ranges. They proved that this framework dissipates, during the fracture process, an amount of energy equal to the fracture energy of the material and that the model is able to propagate the crack with the right speed.

In this paper, novel numerical benchmarks are presented to validate the method in various fracture conditions. The two first ones include an initial notch and study the fracture propagation under two different dynamic loadings (impact and blast). The two other ones focus on the fragmentation of initially unbroken specimens due to uniform expansion in order to demonstrate the ability of the new framework to model crack initiations. Results are in all cases in agreement with the ones reported in the literature.
\end{abstract}

\section{G. Becker}

University of Liège, CM3

Chemin des Chevreuils 1, 4000 Liège, Belgium

Tel.: +32-4-3669552

Fax: +32-4-3669217

E-mail: gauthier.becker@ulg.ac.be

L. Noels

University of Liège, CM3

Chemin des Chevreuils 1, 4000 Liège, Belgium

Tel.: +32-4-3664826

Fax: +32-4-3669217

E-mail: L.Noels@ulg.ac.be
Keywords Discontinuous Galerkin · Cohesive law . parallel implementation $\cdot$ fragmentation $\cdot$ shell

\section{Introduction}

In the past decades, numerical techniques were developed to predict tearing of thin structures. One of them is the combination of the cohesive approach with the finite element method. As a cohesive law, also called "Traction Separation Law" (TSL), models the separation work between the crack lips, cohesive elements can suitably be inserted between two finite elements to integrate the cohesive law and to model crack initiations or propagation between them. Such a cohesive model was pioneered by Hillerborg et al (1976).

Different TSL were proposed in the literature. In order to study the debonding of a spherical inclusion in a ductile matrix, Needleman (1987) suggested having recourse to a polynomial potential law, as depicted on Figure 1(a). As this law only allowed for normal opening, an extension of the polynomial potential cohesive model was supplied by Tvergaard (1990) who suggested considering a uni-dimensional effective opening to model mixed mode fractures. Then as the experiments show a universal exponential shape between the binding energy and the atomistic separation, Needleman (1990a,b) adapted the model and used the exponential cohesive law depicted on Figure 1(b). To account for the dependency of fracture toughness with the plasticity, Tvergaard and Hutchinson (1992) developed a trapezoidal cohesive law restricted to mode I, see Figure 1(c), and Tvergaard and Hutchinson (1993) extended this model to mixed mode fractures. The dwell region introduced in this law is supposed to model the plastic work of the fracture process. In their study they varied the length of the plateau and demonstrated the little effect of the shape of the cohesive law on the results, except for the initial slope, which influence is discussed be- 


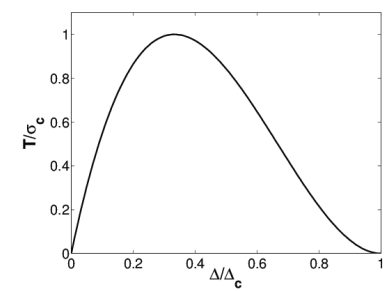

(a) Polynomial (Needleman, 1987).

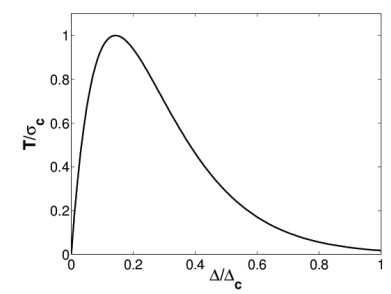

(b) Exponential (Needleman, 1990a).

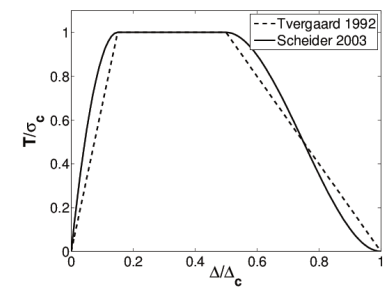

(c) Trapezoidal (Tvergaard and Hutchinson, 1992; Scheider and Brocks, 2003).

Fig. 1 Different (intrinsic) cohesive laws.

low. Scheider and Brocks (2003) modified this law by inserting quadratic and cubic functions in place of linear branches to remove the slope discontinuity at the extremities of the dwell region, see Figure 1(c). With this modified model they successfully simulated the cup cone fracture of a uni-axial tension test in a 2-D axisymmetric setting. However, the nature of ductile fracture cannot be modeled by this cohesive law. Indeed, ductile fracture involves nucleation, growth and coalescence of micro-cavities which cannot be resolved by a cohesive law with constant fracture parameters. To solve this issue Tvergaard and Hutchinson (1996) modified their model and consider a $\sigma_{\mathrm{c}}$ depending on the plastic strain rate. More discussions on how to account for ductility will be given in the Section 4 of this work.

A major problem with the use of cohesive elements is their insertion, which presents some issues. Indeed, if it is performed at the beginning of the simulation, the cohesive law is called intrinsic, and has to model the continuous part of the deformation (Needleman, 1987; Tvergaard, 1990; Tvergaard and Hutchinson, 1993). If this insertion is not limited to pre-defined crack-path, this cannot be realized in a consis- tent way and leads therefore to inaccurate results as shown by Xu and Needleman (1994). On the contrary, the insertion during the simulation, as proposed by Camacho and Ortiz (1996); Ortiz et al (2000), allows using an extrinsic cohesive law but requires mesh modifications. In fact, to insert the cohesive elements the nodes have to be duplicated leading to a very complex implementation, especially in the case of a parallel computation (Pandolfi et al, 2000; Mota et al, 2008). Note that such extrinsic approaches have been developed for shells by Cirak et al (2005).

One method (among others) that can be used to solve these issues is the combination between the discontinuous Galerkin formulation and the extrinsic cohesive law as it has been suggested by Mergheim et al (2004) and demonstrated by Radovitzky et al (2011); Prechtel et al (2011). The discontinuous Galerkin method allows modeling (weakly) the continuum part of the deformation in a consistent way with discontinuous elements. At onset of fracture, this discontinuity can be exploited to insert a cohesive element without mesh modification. Becker and Noels (2011); Becker et al (2011) have extended this combination to thin structures (discretized with beam, plate or shell elements) under the assumption of linear elasticity and more recently for elasto-plastic finite deformations (Becker and Noels, 2012). Note that the use of the DG method has an extra advantage for thin body formulations. Indeed, for shells the continuity of displacement derivatives has also to be ensured. One popular method to enforce this requirement is to insert rotational degrees of freedom. But with the DG method this can be (weakly) achieved through the use of interface terms leading to a one field (displacement) formulation and thus reducing the computational time. This weak enforcement has been previously developed by Engel et al (2002); Hansbo and Larson (2002); Wells and Dung (2007) for linear plates, by Noels and Radovitzky (2008) for linear shells, by Dung and Wells (2008) for geometrically non-linear shells and by Noels (2009) for shells under finite deformation gradients. Error analyzes have also been carried out for general linear bi-harmonic equations by Süli and Mozolevski (2007).

One apparent limit of the methods based on the cohesive element concept is that cracks can only follow a path aligned with the mesh. However Zhou and Molinari (2004a) studied the mesh dependency and concluded that a few randomness in the mesh size and orientation allows significantly reducing the mesh dependency while Papoulia et al (2006) reported the convergence for a pin-wheel based mesh. On top of the convergence of the crack path and crack growth rate, Molinari et al (2007) studied the convergence of the dissipated energy with respect to the mesh size. For meshes with elements of various sizes, a monotonic convergence was achieved for ultra thin meshes.

The XFEM approach is another popular method used in fracture mechanics. It circumvents the requirement of a 
crack to follow the mesh as it allows modeling a crack inside an element. Nevertheless, the DG/ECL framework seems more suitable than the XFEM to investigate large problems or/and problems involving fragmentation as structures subjected to shock, blast... Indeed, the DG/ECL framework can be easily implemented in parallel as the discontinuous nature of the mesh allows its easy partitioning. Besides, to the authors' knowledge only a few 2D fragmentation cases are reported in the XFEM literature, and the cohesive approach is more developed in the fragmentation field. Recently the DG/ECL approach has been used to study fragmentation with 3D elements (Radovitzky et al, 2011; Levy, 2010; Levy et al, 2012).

In this paper, in order to demonstrate its efficiency, the DG/ECL framework for shells is applied to study two different fracture processes. On the one hand, the dynamic crack propagation following impact or blast loading is analyzed in an elastic plate and in an elasto-plastic notched cylinder. In the first example, previously studied by Zavattieri (2006), the impact is simulated by a contact between the plate and a rigid cylinder. The second benchmark was recently presented by Larsson et al (2011) who studied the problem of the blasted-cylinder with the XFEM method and provided experimental data. It is shown that the literature data and the results obtained with the DG/ECL method for shells are in good agreement. On the other hand, the fragmentation process in an initially un-cracked specimen is studied with the aim to demonstrate the ability of the presented framework to model multiple crack initiations. This is achieved by considering the fragmentation of a plate ring under centrifugal forces presented in the literature by Zhou and Molinari (2004a). Furthermore, the expansion of a thin sphere reported in the literature by Levy (2010) is also simulated with the DG/ECL framework using shell elements in place of 3D elements (Zhou and Molinari, 2004a; Levy, 2010; Levy et al, 2012). In this paper it is shown that similar results are found for thin enough bodies. The results obtained for the different applications, elastic or elasto-plastic behaviors, crack propagation or fragmentation dynamics, are in good agreement with the numerical/experimental results of the literature. However, while the published results were relying on different methods (XFEM, embedded discontinuities, cohesive elements ...) in the different references, the presented simulations are conducted with a single computational method, which does not require modifications for the different studied problems. This demonstrates the versatility of the approach. Also, all these simulations are conducted in a scalable parallel environment, which was straightforwardly implemented in an existing finite-element code. This is another advantage of the framework.

The paper is organized as follows. The mechanics of thin bodies is summarized in Section 2. Afterward, the main concepts of the DG formulation of thin bodies are briefly dis- cussed in Section 3. In particular this section demonstrates that the linearization of the general non-linear DG formulation presented by Becker and Noels (2012) leads to the linear formulation developed by Becker et al (2011). Then, Section 4 presents the combined DG/cohesive model specific to thin bodies developed recently by Becker et al (2011); Becker and Noels (2012) for linear and non-linear shells, respectively. Finally, Section 5 presents some numerical benchmarks for crack propagation and for fragmentation problems, for linear and non-linear material behaviors. Numerical results are compared to the ones found in the literature and are shown to be in good agreement.

\section{Mechanics of Kirchhoff-Love shells}

The continuum mechanics of thin bodies is largely discussed in the literature e.g. (Simo and Fox, 1989; Simo et al, 1989, 1990a,b; Simo and Kennedy, 1992; Cirak et al, 2000; Cirak and Ortiz, 2001; Noels and Radovitzky, 2008; Noels, 2009; Becker et al, 2011; Becker and Noels, 2012). Therefore this section regroups only the key concepts of this formulation that are used to obtain the full-discontinuous Galerkin discretization presented in Section 3. In particular, the three last references mentioned display the equations with exactly the same notations that those used herein.

\subsection{Notations}

Hereinafter, a subscript will be used to refer to values formulated in the considered basis, while a superscript will be used to refer to values expressed in the conjugate basis. Roman letters as a subscript or superscript substitute for integers between one and three, while Greek letters substitute for integers one or two. In this paper, the subscript 0 is used to refer to the reference configuration.

Furthermore, the jump $\llbracket \bullet \rrbracket$ and mean $\langle\bullet\rangle$ operators are respectively defined by,

$$
\llbracket \bullet \rrbracket=\bullet^{+}-\bullet^{-} \text {, and }\langle\bullet\rangle=\frac{1}{2}\left(\bullet^{+}+\bullet^{-}\right) \text {. }
$$

\subsection{Kinematics of thin bodies}

The kinematics of thin bodies, as represented on Figure 2, can be described by considering its mid-surface section as a Cosserat plane $\mathscr{A}$ and a third coordinate, representing the thickness, which belongs to the interval $\left[h_{\min } ; h_{\max }\right]$. The thickness $h=h_{\text {max }}-h_{\text {min }}$ of the thin body is assumed to remain small compared to the characteristic lengths in the other directions. In the reference frame $\boldsymbol{E}_{I}$, this representation is written,

$$
\boldsymbol{\xi}=\sum_{I=1}^{3} \xi^{I} \boldsymbol{E}_{I}: \mathscr{A} \times\left[h_{\text {min }} ; h_{\text {max }}\right] \rightarrow \mathbb{R}^{3} \text {. }
$$




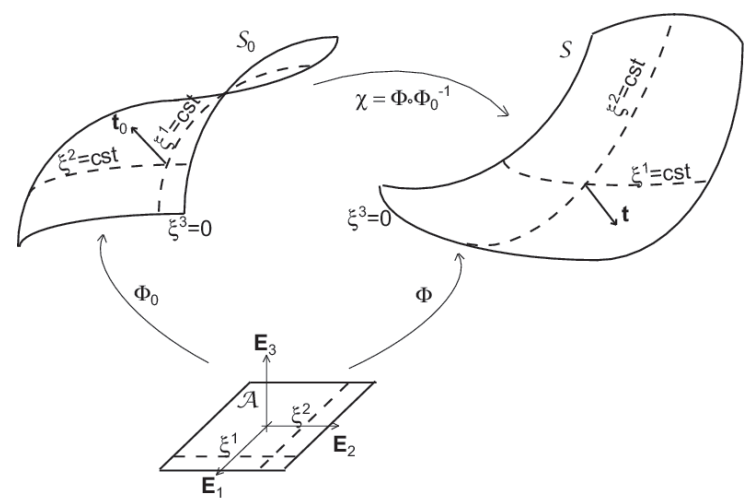

Fig. 2 Description of the different configurations of the shell.

Using $\boldsymbol{\varphi}\left(\xi^{1}, \xi^{2}\right): \mathscr{A} \rightarrow \mathbb{R}^{3}$ the mapping of the mid-surface and $\boldsymbol{t}: \mathscr{A} \rightarrow S^{2}=\left\{\left.\boldsymbol{t} \in \mathbb{R}^{3}\right|_{\|\boldsymbol{t}\|=1}\right\}$ the director of the midsurface, with $S^{2}$ the unit sphere manifold, a configuration $\mathscr{S}$ of the shell is represented by the manifold of position $\boldsymbol{x}$, which is obtained by the mapping $\boldsymbol{\Phi}: \mathscr{A} \times\left[h_{\min } ; h_{\text {max }}\right] \rightarrow$ $\mathscr{S}$

$\boldsymbol{x}=\boldsymbol{\Phi}\left(\xi^{I}\right)=\boldsymbol{\varphi}\left(\xi^{\alpha}\right)+\xi^{3} \lambda_{h} \boldsymbol{t}\left(\xi^{\alpha}\right)$,

where $\lambda_{h}$ is the thickness stretch of the shell. By convention, $\mathscr{S}$ refers to the current configuration of the shell, while the reference configuration $\mathscr{S}_{0}$ is obtained by the mapping $\boldsymbol{\Phi}_{0}=\boldsymbol{\varphi}_{0}\left(\xi^{\alpha}\right)+\xi^{3} \boldsymbol{t}_{0}\left(\xi^{\alpha}\right)$. During the shell deformation, the director of the mid-surface $t$ can be decomposed into the reference director $\boldsymbol{t}_{0}$ and its variation $\boldsymbol{\Delta t}=\boldsymbol{t}-\boldsymbol{t}_{0}$. Moreover, under Kirchhoff-Love assumption one has

$\boldsymbol{t}=\frac{\boldsymbol{\varphi}_{, 1} \wedge \boldsymbol{\varphi}_{, 2}}{\left\|\boldsymbol{\varphi}_{, 1} \wedge \boldsymbol{\varphi}_{, 2}\right\|}$

The two-point deformation gradient between both configurations can be formulated

$\mathbf{F}=\boldsymbol{\varphi}_{, \alpha} \otimes \boldsymbol{g}_{0}^{\alpha}+\xi^{3} \lambda_{h} \boldsymbol{t}_{, \alpha} \otimes \boldsymbol{g}_{0}^{\alpha}+\lambda_{h} \boldsymbol{t} \otimes \boldsymbol{g}_{0}^{3}$,

where,

$\boldsymbol{g}_{\alpha}=\frac{\partial \boldsymbol{\Phi}}{\partial \xi^{\alpha}}=\boldsymbol{\varphi}_{, \alpha}+\xi^{3} \lambda_{h} \boldsymbol{t}_{, \alpha}$, and $\boldsymbol{g}_{3}=\frac{\partial \boldsymbol{\Phi}}{\partial \xi^{3}}=\lambda_{h} \boldsymbol{t}$.

In these relations, the gradient of the variation of thickness, coming from the term $\xi^{3} t \lambda_{h, \alpha}$ has been neglected. Indeed, this gradient of thickness deformation would correspond to a shearing, and can therefore be omitted, since this omission introduces an error of the same order as the Kirchhoff-Love assumption.

Finally, if $j=\operatorname{det}(\nabla \boldsymbol{\Phi})$, and $j_{0}=\operatorname{det}\left(\nabla \boldsymbol{\Phi}_{0}\right)$, the Jacobian of the deformation gradient reads

$J=\operatorname{det}(\mathbf{F})=\frac{j}{j_{0}}$,

with its value in the mid-surface

$\bar{J}=\frac{\bar{j}}{\bar{j}_{0}}$.

\subsection{Governing equations of shells}

The governing equations of a thin body are obtained by integrating on the thickness the equations of force and moment equilibrium, leading to

$$
\begin{aligned}
\bar{\rho} \ddot{\boldsymbol{\varphi}}-\frac{1}{\bar{j}}\left(\bar{j} \boldsymbol{n}^{\alpha}\right)_{, \alpha} & =0 \text { on } \mathscr{A}, \text { and } \\
\frac{1}{\bar{j}}\left(\bar{j} \tilde{\boldsymbol{m}}^{\alpha}\right)_{, \alpha}-\boldsymbol{l}+\lambda \boldsymbol{t} & =0 \text { on } \mathscr{A},
\end{aligned}
$$

where $\lambda$ is an undefined pressure, $\bar{\rho}=h \rho$ is the density by unit of surface, $h$ the thickness and where the Jacobian of the mid-surface is $\bar{j}=\lambda_{h}\left\|\boldsymbol{\varphi}_{, 1} \wedge \boldsymbol{\varphi}_{, 2}\right\|$. Furthermore, the integration on the thickness of the Cauchy stress tensor $\boldsymbol{\sigma}$ (Simo and Fox, 1989; Simo et al, 1989) leads to the definition of

$$
\begin{aligned}
\boldsymbol{n}^{\alpha} & =\frac{1}{\bar{j}} \int_{h_{\min 0}}^{h_{\max 0}} \tau \boldsymbol{g}^{\alpha} \operatorname{det}\left(\boldsymbol{\nabla} \boldsymbol{\Phi}_{0}\right) d \xi^{3} \\
\boldsymbol{m}^{\alpha} & =\frac{\lambda_{h}}{\bar{j}} \boldsymbol{t} \wedge \int_{h_{\min 0}}^{h_{\max 0}} \xi^{3} \boldsymbol{\tau} \boldsymbol{g}^{\alpha} \operatorname{det}\left(\boldsymbol{\nabla} \boldsymbol{\Phi}_{0}\right) d \xi^{3}=\lambda_{h} \boldsymbol{t} \wedge \tilde{\boldsymbol{m}}^{\alpha} \\
\boldsymbol{l} & =\frac{1}{\bar{j}} \int_{h_{\min 0}}^{h_{\max 0}} \tau \boldsymbol{g}^{3} \operatorname{det}\left(\boldsymbol{\nabla} \boldsymbol{\Phi}_{0}\right) d \xi^{3}
\end{aligned}
$$

respectively the resultant stress vector, the resultant torque vector and the resultant across-the-thickness stress vector written in term of the Kirchhoff stress tensor $\boldsymbol{\tau}=J \boldsymbol{\sigma}$. Note that in equations (9) and (10) the inertial angular forces are neglected ${ }^{1}$ and the external forces are omitted for conciseness.

In order to define the stress components, the resultant stress vectors are decomposed in the convected basis, as

$$
\begin{aligned}
\boldsymbol{n}^{\alpha} & =n^{\alpha \beta} \boldsymbol{\varphi}_{, \beta}+q^{\alpha} \lambda_{h} \boldsymbol{t}=\left(\tilde{n}^{\alpha \beta}+\lambda_{\mu}^{\beta} \tilde{m}^{\alpha \mu}\right) \boldsymbol{\varphi}_{\beta}+\lambda_{h} q^{\alpha} \boldsymbol{t}, \\
\tilde{\boldsymbol{m}}^{\alpha} & =\tilde{m}^{\alpha \beta} \boldsymbol{\varphi}_{, \beta}+\tilde{m}^{3 \alpha} \lambda_{h} \boldsymbol{t}, \text { and } \\
\boldsymbol{l} & =l^{\alpha} \boldsymbol{\varphi}_{, \alpha}+l^{3} \lambda_{h} \boldsymbol{t}=\left(\tilde{l}^{\alpha}+\lambda_{\mu}^{\alpha} \tilde{m}^{3 \mu}\right) \boldsymbol{\varphi}_{, \alpha}+l^{3} \lambda_{h} \boldsymbol{t} .
\end{aligned}
$$

In these expressions, $\tilde{n}^{\alpha \beta}$ is the resultant membrane stress, $\tilde{m}^{\alpha \beta}$ is the resultant stress couple, $l^{\alpha}$ is the resultant outof-plane stress, $\tilde{m}^{\alpha 3}$ is the resultant out-of-plane stress couple and $\lambda_{\mu}^{\beta}=\lambda_{h} \boldsymbol{t}_{\mu} \cdot \boldsymbol{\varphi}^{\beta}$ characterizes the curvature of the shell. Due to the symmetry of the Cauchy stress tensor and as $\lambda_{h, \alpha}$ is neglected, one has $q^{\alpha}=l^{\alpha}-\lambda_{\mu}^{\alpha} \tilde{m}^{3 \mu}=\tilde{l}^{\alpha}$, see Noels (2009) for details. Also, $\tilde{l}^{\alpha} \simeq l^{\alpha}$, as $\tilde{m}^{3 \mu}$ vanishes for thin plates.

Under the Kirchhoff-Love shell assumption, $\tilde{l}^{\alpha}$ can be neglected, but it is kept temporarily in the equations in order to develop the full-DG formulation. Indeed, as the components $\boldsymbol{n}^{\alpha}$ and $\tilde{\boldsymbol{m}}^{\alpha}$ of the shell equations do not consider explicitly the normal displacement ${ }^{2}$, equation (16) will allow

1 The inertial angular forces can be neglected if the thickness is sufficiently thin, which is usually the case for thin body formulations.

${ }^{2}$ The normal displacement appears only in $\tilde{\boldsymbol{m}}^{\alpha}$ via its first derivative. 
ensuring that the continuity of the normal deflection across interfaces is weakly satisfied.

This set of governing equations is accompanied by conventional boundary conditions applied on the boundary $\partial \mathscr{A}$ of the mid-surface $\mathscr{A}$, see Noels and Radovitzky (2008) for details.

\subsection{Constitutive behavior}

The previous set of equations is completed by a constitutive relation linking the deformations to the stresses. Herein, depending on the application, two different material laws are considered. On the one hand the well known Hooke's law is used to investigate benchmarks under linear small strains assumption and on the other hand, a $J_{2}$-flow hyperelasticbased law is used for benchmarks exhibiting large deformations and plasticity.

\subsubsection{Hooke's law}

The Hooke's law assumes a linear elastic response between the deformation and stress tensors. Furthermore, this law considers a small displacement field, which leads to,

$$
\begin{aligned}
\boldsymbol{\varphi}_{h} & =\boldsymbol{\varphi}_{0}+\boldsymbol{u}_{h} \text { and } \\
\boldsymbol{t}(\boldsymbol{u}) & =\boldsymbol{t}_{0}+\varepsilon_{\alpha \beta 3}\left[\frac{\boldsymbol{\varphi}_{0, \alpha} \wedge \boldsymbol{u}_{\beta}}{\bar{j}_{0}}+\boldsymbol{t}_{0} \boldsymbol{u}_{, \alpha} \cdot \frac{\boldsymbol{t}_{0} \wedge \boldsymbol{\varphi}_{0, \beta}}{\bar{j}_{0}}\right] .
\end{aligned}
$$

The last relation is a first order approximation of the unit vector, under Kirchhoff-Love assumption, where the symbol $\varepsilon_{\alpha \beta 3}$ is the Levi-Civita permutation tensor. Such an approximation can be used as the second and higher order terms are negligible in the linear range.

For thin bodies the law can be written in plane-stress state by prescribing the value of $\varepsilon_{33}$ in the convected basis (in place of an iterative procedure on the integration points of the thickness, see Section 2.4.2),

$\varepsilon_{33}=-\frac{v}{1-v} \boldsymbol{\varphi}_{0}^{, \alpha} \cdot \boldsymbol{\varphi}_{0}^{, \beta}\left(\frac{1}{2} \boldsymbol{\varphi}_{, \gamma} \cdot \boldsymbol{\varphi}_{, \delta}-\frac{1}{2} \boldsymbol{\varphi}_{0, \gamma} \cdot \boldsymbol{\varphi}_{0, \delta}\right)$.

Since integrated stresses have been decomposed into membrane, shearing and bending stresses acting on the mid-surface convected basis, following Simo and Fox (1989), the deformations are also separated into membrane $\varepsilon$, shearing $\delta$ and torque $\rho$ strain components,

$$
\begin{aligned}
\varepsilon_{\alpha \beta}= & \frac{1}{2} \boldsymbol{\varphi}_{, \alpha} \cdot \boldsymbol{\varphi}_{, \beta}-\frac{1}{2} \boldsymbol{\varphi}_{0, \alpha} \cdot \boldsymbol{\varphi}_{0, \beta} \\
= & \frac{1}{2} \boldsymbol{\varphi}_{0, \alpha} \cdot \boldsymbol{u}_{, \beta}+\frac{1}{2} \boldsymbol{u}_{, \alpha} \cdot \boldsymbol{\varphi}_{0, \beta}, \\
\delta_{\alpha}= & \frac{\boldsymbol{u}_{, \alpha} \cdot \boldsymbol{t}_{0}+\Delta \boldsymbol{t} \cdot \boldsymbol{\varphi}_{0, \alpha}}{2}=0, \text { and } \\
\rho_{\alpha \beta}= & \boldsymbol{\varphi}_{, \alpha} \cdot \boldsymbol{t}_{, \beta}-\boldsymbol{\varphi}_{0, \alpha} \cdot \boldsymbol{t}_{0, \beta} \\
= & \boldsymbol{\varphi}_{0, \alpha \beta} \cdot \boldsymbol{t}_{0} \frac{e_{\mu \eta 3}}{\bar{j}_{0}} \boldsymbol{u}_{, \mu} \cdot\left(\boldsymbol{\varphi}_{0, \eta} \wedge \boldsymbol{t}_{0}\right) \\
& +\frac{e_{\mu \eta 3}}{\bar{j}_{0}} \boldsymbol{u}_{, \mu} \cdot\left(\boldsymbol{\varphi}_{0, \alpha \beta} \wedge \boldsymbol{\varphi}_{0, \eta}\right)-\boldsymbol{u}_{, \alpha \beta} \cdot \boldsymbol{t}_{0} .
\end{aligned}
$$

The elastic constitutive relations between the effective (linearized) stresses and strains read, (Simo and Fox, 1989)

$$
\begin{aligned}
\tilde{n}^{\alpha \beta} & =\frac{E\left(h_{\max }-h_{\min }\right)}{1-v^{2}} \mathscr{H}^{\alpha \beta \gamma \delta} \varepsilon_{\gamma \delta}=\mathscr{H}_{n}^{\alpha \beta \gamma \delta} \varepsilon_{\gamma \delta}, \\
\tilde{m}^{\alpha \beta} & =\frac{E\left(h_{\max }-h_{\min }\right)^{3}}{12\left(1-v^{2}\right)} \mathscr{H}^{\alpha \beta \gamma \delta} \rho_{\gamma \delta}=\mathscr{H}_{m}^{\alpha \beta \gamma \delta} \rho_{\gamma \delta}, \text { and } \\
\tilde{l}^{\alpha} & =G\left(h_{\max }-h_{\min }\right) \frac{A^{\prime}}{A} \mathscr{H}^{\alpha \beta} \gamma_{\beta}=\mathscr{H}_{s}^{\alpha \beta} \gamma_{\beta},
\end{aligned}
$$

where $E$ is the Young modulus, $v$ is the Poisson's ration, $G$ is the shear modulus, $\gamma=2 \delta$ and where

$$
\begin{aligned}
\mathscr{H}^{\alpha \beta \gamma \delta}= & v \boldsymbol{\varphi}_{0}^{\alpha} \cdot \boldsymbol{\varphi}_{0}^{, \beta} \boldsymbol{\varphi}_{0}^{, \gamma} \cdot \boldsymbol{\varphi}_{0}^{, \delta}+\frac{1}{2}(1-v) \\
& {\left[\boldsymbol{\varphi}_{0}^{, \alpha} \cdot \boldsymbol{\varphi}_{0}^{, \gamma} \boldsymbol{\varphi}_{0}^{, \delta} \cdot \boldsymbol{\varphi}_{0}^{, \beta}+\boldsymbol{\varphi}_{0}^{, \alpha} \cdot \boldsymbol{\varphi}_{0}^{, \delta} \boldsymbol{\varphi}_{0}^{, \gamma} \cdot \boldsymbol{\varphi}_{0}^{, \beta}\right], } \\
\mathscr{H}^{\alpha \beta}= & \boldsymbol{\varphi}_{0}^{, \alpha} \cdot \boldsymbol{\varphi}_{0}^{, \beta} .
\end{aligned}
$$

In these last expressions $\boldsymbol{\varphi}_{0}^{, \alpha}$ denotes, with an abuse of notation, the conjugate basis to $\boldsymbol{\varphi}_{0, \alpha}$.

\subsection{2 $J_{2}$-flow hyperelastic-based law}

The plastic behavior of the material is taken into account by the $J_{2}$-flow theory with an isotropic linear hardening. The model is based on an hyperelastic formulation, which implies the assumption of a multiplicative decomposition of the finite deformation gradient $\mathbf{F}$ into an elastic part $\mathbf{F}^{e}$ and a plastic part $\mathbf{F}^{p}$. Thus, the stress tensor derives from an elastic potential $W$, which, in the bi-logarithmic form, reads

$W\left(\mathbf{C}^{e}\right)=\frac{K}{2} \log J^{2}+\frac{G}{4}\left[\log \mathbf{C}^{e}\right]^{\mathrm{dev}}:\left[\log \mathbf{C}^{e}\right]^{\mathrm{dev}}$,

with $K$ and $G$ respectively the bulk and shear moduli. As $W$ should only depend on the elastic deformations, the elastic right Cauchy strain tensor, defined by $\mathbf{C}^{e}=\left(\mathbf{F}^{e}\right)^{T} \mathbf{F}^{e}$, is considered in (28). Using these definitions the first PiolaKirchhoff stress tensor follows from

$\mathbf{P}=2 \mathbf{F} \cdot\left[\left(\mathbf{F}^{p}\right)^{-1} \frac{\partial W\left(\mathbf{C}^{e}\right)}{\partial \mathbf{C}^{e}}\left(\mathbf{F}^{p}\right)^{-T}\right]$.

Then, the incremental theory can be used to determine the stresses at stage $n+1$ from the known values at stage $n$ as it is described in Cuitino and Ortiz (1992); Deiterding et al (2006).

Finally, the determination of the thickness stretch $\lambda_{h}$, required to evaluate the deformation gradient (5) has to be specified. The thickness is discretized with 11 integration points following a Simpson integration rule. Then, the local value $\lambda_{h}^{p}$ is determined at each point, using NewtonRaphson iterations, to satisfy locally the plane stress requirement $\tau^{33}=0$. To achieve this, the Kirchhoff stress tensor $\boldsymbol{\tau}=\mathbf{P F}^{T}$ is expressed in the convected basis thanks to

$\tau^{i j}=\tau_{i j} \boldsymbol{g}^{i} \otimes \boldsymbol{g}^{j}$,

which can be used to compute Eqs. $(11$ - 13). Finally, the global thickness stretch $\lambda_{h}$ is then determined from the Simpson integration on the 11 local values $\lambda_{h}^{p}$. 


\section{Discontinuous Galerkin formulation}

The system of Eqs. (9-10) can be solved using the finite element method. This weak form of the system considers a discretization of $\mathscr{A}$ into elements $\mathscr{A}_{e}$. Furthermore, instead of seeking the exact solution $\boldsymbol{\varphi}$, a polynomial approximation $\boldsymbol{\varphi}_{h}$ constitutes the solution of the finite element problem. In continuous Galerkin formulation, this approximation is continuous from one element to its neighbors ensuring in this way the continuity of the solution. Moreover, for thin bodies, the continuity of the first derivative of $\boldsymbol{\varphi}$ has also to be verified. However, the traditional Lagrangian interpolation does not satisfy this $C^{1}$ requirement, but $C^{1}$ continuity can be ensured weakly within a discontinuous Galerkin framework. This weak enforcement leads to a one-field (displacement) formulation (Engel et al, 2002; Hansbo and Larson, 2002; Wells and Dung, 2007; Noels and Radovitzky, 2008; Dung and Wells, 2008; Noels, 2009). Recently, with the aim of taking into account initiations and propagation of fracture, this formulation was extended to discontinuous polynomial approximations for linear beams by Becker and Noels (2011), linear shells by Becker and Noels (2011) and nonlinear shells by Becker and Noels (2012). This paper summarizes these developments for non-linear and linear shells. In particular, the weak form is first obtained for the nonlinear case and it is shown that its linearization results in the linear formulation presented by Becker et al (2011).

\subsection{Weak form of the non-linear problem}

Let us consider a discretization of $\mathscr{A}$ into finite elements $\mathscr{A}_{e}$. The discontinuous Galerkin framework is based on finding an approximation $\boldsymbol{\varphi}_{h}$ of the exact solution $\boldsymbol{\varphi}$, which belongs in the manifold $U_{h}=\left\{\left.\boldsymbol{\varphi}_{h} \in \mathbf{L}^{2}(\mathscr{A})\right|_{\boldsymbol{\varphi}_{h} \in \mathbb{P}^{k}\left(\mathscr{A}_{e}\right)}\right\} \subset$ $\prod_{e} \mathbf{H}^{2}\left(\mathscr{A}_{e}\right)$, where $k \geq 2$ is the degree of the polynomial approximation in a finite element.

Due to the discontinuities arising from the definition of $U_{h}$, discontinuous Galerkin methods usually introduce three interfaces terms

1. The consistency term results directly from the discontinuous approximation of the solution having jumps between two elements.

2. The symmetrization term is introduced to ensure a symmetric formulation (in the linear range).

3. The stability term is introduced to ensure compatibility and stability as it is well known that for elliptic problems a discontinuous Galerkin formulation leads to instabilities. As for the symmetrization term, the introduction of this term does not modify the consistency of the method. The stability term depends on a stability parameter which is independent of the mesh size and of the material properties.
Fig. 3 Local basis vector on an interface element tangential to the shell. The interface is drawn with dotted line. By convention, for the interface element, $\boldsymbol{\varphi}_{, 1}$ is parallel and $\boldsymbol{\varphi}_{, 2}$ is normal to the interface.

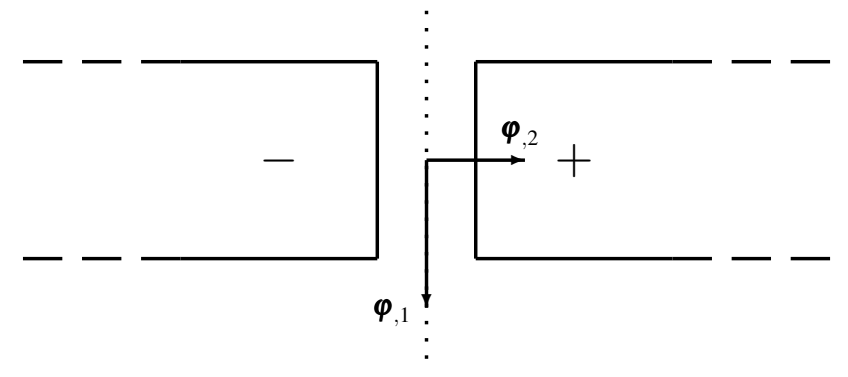

These 3 terms can be developed to ensure weakly the $C^{1}$ continuity for a non-linear finite element discretization of shells, as presented by Noels (2009)

$$
\begin{aligned}
a_{m I 1}^{s}\left(\boldsymbol{\varphi}_{h}, \delta \boldsymbol{\varphi}\right)= & \int_{s}\left\langle\bar{j} \lambda_{h} \tilde{\boldsymbol{m}}^{\alpha}\right\rangle \cdot \llbracket \delta \boldsymbol{t} \rrbracket v_{\alpha}^{-} d \partial \mathscr{A}_{e}, \\
a_{m I 2}^{s}\left(\boldsymbol{\varphi}_{h}, \delta \boldsymbol{\varphi}\right)= & \int_{s} \llbracket \boldsymbol{t} \rrbracket \cdot\left\langle\bar{j} \lambda_{h} \tilde{\boldsymbol{m}}^{\alpha} \cdot \boldsymbol{\varphi}_{, \beta} \delta \boldsymbol{\varphi}_{, \beta}+\bar{j}_{0} \mathscr{H}_{m}^{\alpha \beta \gamma \delta}\right. \\
& \left.\left(\delta \boldsymbol{\varphi}_{, \gamma} \cdot \boldsymbol{t}_{, \delta}+\boldsymbol{\varphi}_{, \gamma} \cdot \delta \boldsymbol{t}_{, \delta}\right) \boldsymbol{\varphi}_{, \beta}\right\rangle v_{\alpha}^{-} d \partial \mathscr{A}_{e} \\
a_{m I 3}^{s}\left(\boldsymbol{\varphi}_{h}, \delta \boldsymbol{\varphi}\right)= & \int_{s} \llbracket \boldsymbol{t}\left(\boldsymbol{\varphi}_{h}\right) \rrbracket \cdot \boldsymbol{\varphi}_{, \beta} \\
& \left\langle\frac{\beta_{1} \mathscr{H}_{m}^{\alpha \beta \gamma \delta} \overline{j_{0}}}{h^{s}}\right\rangle \llbracket \delta \boldsymbol{t} \rrbracket \cdot \boldsymbol{\varphi}_{, \gamma} v_{\alpha}^{-} v_{\delta}^{-} d \partial \mathscr{A}_{e},
\end{aligned}
$$

which are respectively the consistency (31), symmetrization (32), and stability (33) terms. In the last equation, $\beta_{1}$ is the dimensionless stability parameter, $h^{s}$ is the characteristic size of the element, $\mathscr{H}_{m}$ is the linearized bending stiffness defined in Eq. (24).

In Eqs. (31 - 33), the solution is independent of the choice of the minus and plus element due to the combination of the jump with the outward unit normal of the minus element $v^{-}$, which can be computed by,

$v_{\alpha}^{-}=\boldsymbol{\varphi}_{, 2} \cdot \boldsymbol{\varphi}^{\alpha}$,

where by choice $\boldsymbol{\varphi}_{, 2}$ is the normal of the interface element, as illustrated on Figure 3.

It has to be mentioned that, even in the non-linear range, linear expressions are used for the symmetrization (32) and stability (33) terms. Indeed, these terms are introduced to ensure continuity and stability of the solution and any expressions can be used as long as the consistency is not modified. The use of linearized expressions allows, on the one hand, reducing the computational cost and on the other hand preventing a lack of stability for some materials. Indeed, if the stability term would depend on the tangent moduli in place of the Young modulus, it could vanish for a perfectly plastic constitutive behavior. 
In order to enforce weakly the $C^{0}$ continuity in the context of the full-discontinuous Galerkin framework, additional interface terms are introduced in the non-linear case following exactly the same argumentation developed by Becker and Noels (2012), yielding

$$
\begin{aligned}
& a_{n I 1}^{s}\left(\boldsymbol{\varphi}_{h}, \boldsymbol{\delta} \boldsymbol{\varphi}\right)=\int_{s}\left\langle\bar{j} \boldsymbol{n}^{\alpha}\right\rangle \cdot \llbracket \delta \boldsymbol{\varphi} \rrbracket v_{\alpha}^{-} d \partial \mathscr{A}_{e}, \\
& a_{n I 2}^{s}\left(\boldsymbol{\varphi}_{h}, \boldsymbol{\delta} \boldsymbol{\varphi}\right)=\int_{s} \llbracket \boldsymbol{\varphi}_{h} \rrbracket \cdot\left\langle\boldsymbol{\delta}\left(\bar{j} \boldsymbol{n}^{\alpha}\right)\right\rangle v_{\alpha}^{-} d \partial \mathscr{A}_{e}, \\
& a_{n I 3}^{s}\left(\boldsymbol{\varphi}_{h}, \boldsymbol{\delta} \boldsymbol{\varphi}\right)=\int_{s} \llbracket \boldsymbol{\varphi}_{h} \rrbracket \cdot \boldsymbol{\varphi}_{, \gamma} v_{\delta}^{-}\left\langle\frac{\beta_{2} \mathscr{H}_{n}^{\alpha \beta \gamma \delta} \bar{j}_{0}}{h^{s}}\right\rangle
\end{aligned}
$$$$
\llbracket \delta \varphi \rrbracket \cdot \varphi_{\beta} v_{\alpha}^{-} d \partial \mathscr{A}_{e} \text {, and }
$$

$$
a_{s I 3}^{s}\left(\boldsymbol{\varphi}_{h}, \delta \boldsymbol{\varphi}\right)=\int_{s} \llbracket \boldsymbol{\varphi}_{h} \rrbracket \cdot \boldsymbol{t} v_{\beta}^{-}\left\langle\frac{\beta_{3} \mathscr{H}_{q}^{\alpha \beta} \bar{j}_{0}}{h^{s}}\right\rangle
$$

$$
\llbracket \delta \varphi \rrbracket \cdot t v_{\alpha}^{-} d \partial \mathscr{A}_{e},
$$

respectively the consistency membrane (35), the symmetrization membrane (36), the stability membrane (37) and the stability shearing (38) terms. In these equations $\beta_{2}$ and $\beta_{3}$ are dimensionless stability parameters and $\mathscr{H}_{n}$ and $\mathscr{H}_{q}$ are respectively the linearized membrane and shearing stiffness defined in Eqs. (23) and (25).

The 3 first terms (35-37) ensure a consistent and stable continuity in the Cosserat plane of the mid-surface of the shell. The last one (38) guarantees the continuity of the outof-plane displacement. As lengthy described by Becker and Noels $(2011,2012)$ this expression is obtained by considering the shearing components in the development of the equations. As the Kirchhoff-Love assumption corresponds to neglecting shearing, the related consistency and symmetrization terms can be neglected in such a way that only the stability term remains. The linearized expression of $\delta\left(\bar{j} n^{\alpha}\right)$ in (36) requires some developments similar to the linearization of $\delta\left(\bar{j} \tilde{\boldsymbol{m}}^{\alpha}\right)$ performed in (32), which leads to Becker and Noels (2012),

$$
\begin{aligned}
\boldsymbol{\delta}\left(\bar{j}^{\alpha}\right) & =\frac{\bar{j}_{0}}{2} \mathscr{H}_{n}^{\alpha \beta \gamma \delta}\left(\delta \boldsymbol{\varphi}_{, \gamma} \cdot \boldsymbol{\varphi}_{, \delta}+\boldsymbol{\varphi}_{, \gamma} \cdot \delta \boldsymbol{\varphi}_{, \delta}\right) \boldsymbol{\varphi}_{, \beta} \\
& +\bar{j}^{\alpha} \cdot \boldsymbol{\varphi}^{, \beta} \delta \boldsymbol{\varphi}_{, \beta} \\
& +\frac{\bar{j}_{0}}{\lambda_{h}} \lambda_{\mu}^{\beta} \mathscr{H}_{m}^{\alpha \mu \gamma \delta}\left(\delta \boldsymbol{\varphi}_{, \gamma} \cdot \boldsymbol{t}_{, \delta}+\boldsymbol{\varphi}_{, \gamma} \cdot \delta \boldsymbol{t}_{, \delta}\right) \boldsymbol{\varphi}_{, \beta} \\
& +\bar{j} \lambda_{h} \tilde{m}^{\alpha \mu}\left(\delta \boldsymbol{t}_{, \mu} \cdot \boldsymbol{\varphi}^{, \beta}-\frac{\lambda_{\mu}^{\zeta}}{\lambda_{h}} \boldsymbol{\varphi}^{, \beta} \cdot \delta \boldsymbol{\varphi}_{, \zeta}\right) \boldsymbol{\varphi}_{, \beta}
\end{aligned}
$$

Finally, the continuity of the displacement field is ensured weakly by the seven interface terms given by equations (31-33) and (35-38). These expressions can be used to write the weak form of the problem, see Becker and Noels
(2012) for more details,

$$
\begin{aligned}
a_{d}^{e}\left(\boldsymbol{\varphi}_{h}, \delta \boldsymbol{\varphi}\right)= & -\sum_{e} a_{n}^{e}\left(\boldsymbol{\varphi}_{h}, \delta \boldsymbol{\varphi}\right)-\sum_{e} a_{m}^{e}\left(\boldsymbol{\varphi}_{h}, \delta \boldsymbol{\varphi}\right) \\
& -\sum_{s} a_{n I 1}^{s}\left(\boldsymbol{\varphi}_{h}, \delta \boldsymbol{\varphi}\right)-\sum_{s} a_{m I 1}^{s}\left(\boldsymbol{\varphi}_{h}, \delta \boldsymbol{\varphi}\right) \\
& -\sum_{s} a_{n I 2}^{s}\left(\boldsymbol{\varphi}_{h}, \delta \boldsymbol{\varphi}\right)-\sum_{s} a_{m I 2}^{s}\left(\boldsymbol{\varphi}_{h}, \delta \boldsymbol{\varphi}\right) \\
& -\sum_{s} a_{n I 3}^{s}\left(\boldsymbol{\varphi}_{h}, \delta \boldsymbol{\varphi}\right)-\sum_{s} a_{m I 3}^{s}\left(\boldsymbol{\varphi}_{h}, \delta \boldsymbol{\varphi}\right) \\
& +\sum_{s} a_{s I 3}^{s}\left(\boldsymbol{\varphi}_{h}, \delta \boldsymbol{\varphi}\right),
\end{aligned}
$$

with the traditional inertial, membrane and bending bulk components arising from the shell theory,

$$
\begin{aligned}
& a_{d}^{e}\left(\boldsymbol{\varphi}_{h}, \boldsymbol{\delta} \boldsymbol{\varphi}\right)=\int_{\mathscr{A}_{e}} \bar{\rho} \ddot{\boldsymbol{\varphi}} \cdot \boldsymbol{\delta} \boldsymbol{\varphi} d \mathscr{A}, \\
& a_{n}^{e}\left(\boldsymbol{\varphi}_{h}, \boldsymbol{\delta} \boldsymbol{\varphi}\right)=\int_{\mathscr{A}_{e}} \bar{j} \boldsymbol{n}^{\alpha}\left(\boldsymbol{\varphi}_{h}\right) \cdot \delta \boldsymbol{\varphi}_{, \alpha} d \mathscr{A}, \text { and }, \\
& a_{m}^{e}\left(\boldsymbol{\varphi}_{h}, \boldsymbol{\delta} \boldsymbol{\varphi}\right)=\int_{\mathscr{A}_{e}} \bar{j} \tilde{\boldsymbol{m}}^{\alpha}\left(\boldsymbol{\varphi}_{h}\right) \cdot \boldsymbol{\delta}\left(\lambda_{h} \boldsymbol{t}_{, \alpha}\right) d \mathscr{A} .
\end{aligned}
$$

\subsection{Weak form of the linear problem}

The general non linear weak form (40) can be linearized to obtain the linear full-DG formulation earlier presented by Becker et al (2011), using:

(i) The small displacement field given by Eqs. (17 - 18).

(ii) The linear expression of the resultant stress membrane $\tilde{n}^{\alpha \beta}$ and of the resultant stress couple $\tilde{m}^{\alpha \beta}$ given by Eqs. (23 - 24).

(iii) A first order approximation (i.e. the second and higher order terms are neglected).

Therefore the different terms of equation (40) can be successively linearized as,

$$
\begin{aligned}
a_{l d}^{e}\left(\boldsymbol{\varphi}_{h}, \boldsymbol{\delta} \boldsymbol{\varphi}\right)= & \int_{\mathscr{A}_{e}} \bar{\rho} \ddot{\boldsymbol{u}}_{h} \cdot \boldsymbol{\delta} \boldsymbol{u} d \mathscr{A}, \\
a_{l n}^{e}\left(\boldsymbol{\varphi}_{h}, \boldsymbol{\delta} \boldsymbol{\varphi}\right)= & \int_{\mathscr{A}_{e}} \overline{\bar{j}_{0}} \frac{1}{4}\left(\boldsymbol{\varphi}_{0, \gamma} \cdot \boldsymbol{u}_{h, \delta}+\boldsymbol{\varphi}_{0, \delta} \cdot \boldsymbol{u}_{h, \gamma}\right) \\
& \mathscr{H}_{n}^{\alpha \beta \gamma \delta}\left(\boldsymbol{\varphi}_{0, \beta} \delta \boldsymbol{u}_{, \alpha}+\boldsymbol{\varphi}_{0, \alpha} \delta \boldsymbol{u}_{, \beta}\right) d \mathscr{A} \\
& +\int_{\mathscr{A}_{e}} \bar{j}_{0}\left(\boldsymbol{u}_{h, \gamma} \cdot \boldsymbol{t}_{0, \delta}+\boldsymbol{\varphi}_{0, \gamma} \cdot \boldsymbol{\Delta} \boldsymbol{t}_{, \delta}\right) \\
& \mathscr{H}_{m}^{\alpha \beta \gamma \delta} \boldsymbol{t}_{0, \beta} \cdot \delta \boldsymbol{u}_{, \alpha} d \mathscr{A}, \\
a_{l m}^{e}\left(\boldsymbol{\varphi}_{h}, \boldsymbol{\delta} \boldsymbol{\varphi}\right)= & \int_{\mathscr{A}_{h}} \bar{j}_{0}\left(\boldsymbol{u}_{h, \gamma} \cdot \boldsymbol{t}_{0, \delta}+\boldsymbol{\varphi}_{0, \gamma} \cdot \boldsymbol{\Delta} \boldsymbol{t}_{, \delta}\right) \\
& \mathscr{H}_{m}^{\alpha \beta \gamma \delta} \boldsymbol{\varphi}_{0, \beta} \cdot \boldsymbol{\delta} \boldsymbol{\Delta} \boldsymbol{t}_{, \alpha} d \mathscr{A},
\end{aligned}
$$

$$
\begin{aligned}
a_{l n I 1}^{s}\left(\boldsymbol{\varphi}_{h}, \boldsymbol{\delta} \boldsymbol{\varphi}\right)= & \int_{s} \frac{1}{2}\left\langle\bar{j}_{0} \mathscr{H}_{n}^{\alpha \beta \gamma \delta}\left(\boldsymbol{\varphi}_{0, \gamma} \cdot \boldsymbol{u}_{h, \delta}+\boldsymbol{\varphi}_{0, \delta} \cdot \boldsymbol{u}_{h, \gamma}\right)\right. \\
& \left.+\lambda_{\mu}^{\beta} \bar{j}_{0} \mathscr{H}_{m}^{\alpha \beta \gamma \delta}\left(\boldsymbol{\varphi}_{0, \gamma} \cdot \Delta \boldsymbol{t}_{, \delta}+\boldsymbol{u}_{h, \gamma} \cdot \boldsymbol{t}_{0, \delta}\right)\right\rangle \\
& \boldsymbol{\varphi}_{0, \beta} \cdot \llbracket \delta \boldsymbol{u} \rrbracket v_{\alpha}^{-} d \partial \mathscr{A}_{e},
\end{aligned}
$$




$$
\begin{aligned}
a_{l m I 1}^{s}\left(\boldsymbol{\varphi}_{h}, \delta \boldsymbol{\varphi}\right)= & \int_{s} \llbracket \delta \boldsymbol{\Delta} \boldsymbol{t} \rrbracket \cdot\left\langle\overline { j } _ { 0 } \mathscr { H } _ { m } ^ { \alpha \beta \gamma \delta } \left(\boldsymbol{u}_{h, \gamma} \cdot \boldsymbol{t}_{0, \delta}+\right.\right. \\
& \left.\left.\boldsymbol{\varphi}_{0, \gamma} \cdot \boldsymbol{\Delta} \boldsymbol{t}, \delta\right) \boldsymbol{\varphi}_{0, \beta}\right\rangle v_{\alpha}^{-} d \partial \mathscr{A}_{e}, \\
a_{l n I 2}^{s}\left(\boldsymbol{\varphi}_{h}, \boldsymbol{\delta} \boldsymbol{\varphi}\right)= & \int_{s} \llbracket \boldsymbol{u}_{h} \rrbracket \cdot\left\langle\overline { j } _ { 0 } \boldsymbol { \varphi } _ { 0 , \beta } \left[\frac{1}{2} \mathscr{H}_{n}^{\alpha \beta \gamma \delta}\right.\right. \\
& \left(\delta \boldsymbol{u}_{, \gamma} \cdot \boldsymbol{\varphi}_{0, \delta}+\boldsymbol{\varphi}_{0, \gamma} \cdot \delta \boldsymbol{u}_{, \delta}\right)+\lambda_{\mu}^{\beta} \mathscr{H}_{m}^{\alpha \beta \gamma \delta} \\
& \left.\left.\left(\delta \boldsymbol{u}_{, \gamma} \cdot \boldsymbol{t}_{0, \delta}+\boldsymbol{\varphi}_{0, \gamma} \cdot \boldsymbol{\delta} \Delta \boldsymbol{t}_{, \delta}\right)\right]\right\rangle v_{\alpha}^{-} d \partial \mathscr{A}_{e},
\end{aligned}
$$

$$
\begin{aligned}
a_{l m I 2}^{s}\left(\boldsymbol{\varphi}_{h}, \delta \boldsymbol{\varphi}\right)= & \int_{s} \llbracket \boldsymbol{\Delta} \boldsymbol{t} \rrbracket \cdot\left\langle\bar{j}_{0} \boldsymbol{\varphi}_{0, \beta} \mathscr{H}_{m}^{\alpha \beta \gamma \delta}\right. \\
& \left.\left(\delta \boldsymbol{u}_{, \gamma} \cdot \boldsymbol{t}_{0, \delta}+\boldsymbol{\varphi}_{0, \gamma} \cdot \boldsymbol{\delta} \boldsymbol{\Delta} \boldsymbol{t}_{, \delta}\right)\right\rangle v_{\alpha}^{-} d \partial \mathscr{A}_{e}
\end{aligned}
$$

$$
\begin{aligned}
a_{l n I 3}^{s}\left(\boldsymbol{\varphi}_{h}, \delta \boldsymbol{\varphi}\right)= & \int_{s} \llbracket \boldsymbol{u}_{h} \rrbracket \cdot \boldsymbol{\varphi}_{0, \gamma} v_{\delta}^{-}\left\langle\frac{\beta_{2} \mathscr{H}_{n}^{\alpha \beta \gamma \delta} \bar{j}_{0}}{h^{s}}\right\rangle \\
& \llbracket \delta \boldsymbol{u} \rrbracket \cdot \boldsymbol{\varphi}_{0, \beta} v_{\alpha}^{-} d \partial \mathscr{A}_{e}
\end{aligned}
$$

$$
\begin{aligned}
a_{l m l 3}^{s}\left(\boldsymbol{\varphi}_{h}, \delta \boldsymbol{\varphi}\right)= & \int_{s} \llbracket \boldsymbol{\Delta} \boldsymbol{t} \rrbracket \cdot \boldsymbol{\varphi}_{0, \beta}\left\langle\frac{\beta_{1} \bar{j}_{0} \mathscr{H}_{m}^{\alpha \beta \gamma \delta}}{h^{s}}\right\rangle \\
& \llbracket \delta \boldsymbol{\Delta} \boldsymbol{\Delta} \rrbracket \cdot \boldsymbol{\varphi}_{0, \gamma} v_{\alpha}^{-} v_{\delta}^{-} d \partial \mathscr{A}_{e},
\end{aligned}
$$

$$
a_{l s I 3}^{s}\left(\boldsymbol{\varphi}_{h}, \delta \boldsymbol{\varphi}\right)=\int_{s} \llbracket \boldsymbol{u}_{h} \rrbracket \cdot \boldsymbol{t}_{0} v_{\beta}^{-}\left\langle\frac{\beta_{3} \mathscr{H}_{q}^{\alpha \beta}}{h^{s}}\right\rangle
$$

$$
\llbracket \delta \boldsymbol{u} \rrbracket \cdot \boldsymbol{t}_{0} v_{\alpha}^{-} d \partial \mathscr{A}_{e} .
$$

In the stability expressions (51-53), the effect of curvature $\lambda_{\mu}^{\beta}$ has been neglected without compromising the stability of the method (Becker et al, 2011). These developments allow writing Eq. (40) as,

$$
\begin{aligned}
a_{l d}^{e}\left(\boldsymbol{\varphi}_{h}, \delta \boldsymbol{\varphi}\right)= & -\sum_{e} a_{l n}^{e}\left(\boldsymbol{\varphi}_{h}, \delta \boldsymbol{\varphi}\right)-\sum_{e} a_{l m}^{e}\left(\boldsymbol{\varphi}_{h}, \delta \boldsymbol{\varphi}\right) \\
& -\sum_{s} a_{l n I 1}^{s}\left(\boldsymbol{\varphi}_{h}, \delta \boldsymbol{\varphi}\right)-\sum_{s} a_{l m I 1}^{s}\left(\boldsymbol{\varphi}_{h}, \delta \boldsymbol{\varphi}\right) \\
& -\sum_{s} a_{l n I 2}^{s}\left(\boldsymbol{\varphi}_{h}, \delta \boldsymbol{\varphi}\right)-\sum_{s} a_{l m I 2}^{s}\left(\boldsymbol{\varphi}_{h}, \delta \boldsymbol{\varphi}\right) \\
& -\sum_{s} a_{l n I 3}^{s}\left(\boldsymbol{\varphi}_{h}, \delta \boldsymbol{\varphi}\right)-\sum_{s} a_{l m I 3}^{s}\left(\boldsymbol{\varphi}_{h}, \delta \boldsymbol{\varphi}\right) \\
& +\sum_{s} a_{l s I 3}^{s}\left(\boldsymbol{\varphi}_{h}, \delta \boldsymbol{\varphi}\right),
\end{aligned}
$$

which corresponds to the linear full-discontinuous Galerkin formulation presented in Becker et al (2011). Therefore it is assumed that the non-linear formulation (40) inherits from the numerical properties (convergence and stability) of its linear counterpart. Indeed, Becker et al (2011) demonstrated the stability and convergence of the linearized equations. Thus, the stability is ensured if the parameters $\beta_{1}$ and $\beta_{2}$ are large enough, if $\beta_{3}$ is non-zero. As it is lengthy discussed by Becker and Noels (2011) for linear beams, in order to reduce locking and to preserve stability, the optimal value for $\beta_{3}$ is $\beta_{1} \times\left(\frac{h}{L_{c}}\right)^{2}$, where $h$ is the shell thickness and where $L_{c}$ is a characteristic length depending on the problem. The convergence rate of the method in the energy norm with respect to the mesh size is proved to be equal to $k-1$, with $k$ the degree of the polynomial approximation. Finally, the method presents an optimal-convergence rate $k+1$ in the $\mathrm{L}^{2}$-norm, which can be demonstrated for at least cubic elements.

\section{Fracture model}

The main advantage of a discontinuous Galerkin formulation is its ability to be extended to account for fracture mechanics. Indeed the presented framework can be coupled with an extrinsic cohesive law in a consistent and efficient way as no topological mesh modification is required to propagate a crack. In a recent paper Becker et al (2011) presented a novel cohesive law dedicated to thin body formulations that can be coupled to the full-DG framework presented in Section 3.

The combination between the full-DG formulation and an extrinsic cohesive law can be realized very easily by introducing the cohesive force in equation (40), leading to

$$
\begin{aligned}
0= & \sum_{e}\left(a_{d}^{e}\left(\boldsymbol{\varphi}_{h}, \delta \boldsymbol{\varphi}\right)+a_{n}^{e}\left(\boldsymbol{\varphi}_{h}, \delta \boldsymbol{\varphi}\right)+a_{m}^{e}\left(\boldsymbol{\varphi}_{h}, \delta \boldsymbol{\varphi}\right)\right)+ \\
& \sum_{s}\left[( 1 - \alpha _ { s } ) \left(a_{n I}^{s}\left(\boldsymbol{\varphi}_{h}, \delta \boldsymbol{\varphi}\right)+a_{m I}^{s}\left(\boldsymbol{\varphi}_{h}, \delta \boldsymbol{\varphi}\right)-\right.\right. \\
& \left.\left.a_{s I}^{s}\left(\boldsymbol{\varphi}_{h}, \delta \boldsymbol{\varphi}\right)\right)+\alpha_{s} a_{\text {cohesive }}^{s}\left(\llbracket \boldsymbol{\varphi}_{h} \rrbracket, \llbracket \delta \boldsymbol{\varphi} \rrbracket\right)\right],
\end{aligned}
$$

where $a_{\text {cohesive }}^{s}\left(\llbracket \boldsymbol{\varphi}_{h} \rrbracket, \llbracket \delta \boldsymbol{\varphi} \rrbracket\right)$ is the contribution of the cohesive law that has to be defined, where $\alpha_{s}$ is a Boolean value equal to 0 before fracture initiation and 1 after, and where interface terms account for the three contributions previously defined. The Boolean $\alpha_{s}$ is computed at each Gauss integration point along the interface in such a way that the whole interface element is not necessarily broken as a whole.

A criterion used by Corigliano et al (2008); Molinari et al (2007); Li and Chandra (2003); Pandolfi and Ortiz (2002); Papoulia et al (2003); Zavattieri (2004); Zhang et al (2007); Becker et al (2011); Becker and Noels (2012) to determine fracture onset, is the criterion of Camacho and Ortiz (1996) based on the evaluation of an effective stress,

$\sigma_{\text {eff }}=\left\{\begin{array}{ll}\sqrt{\sigma^{2}+\beta^{-2} \tau^{2}} & \text { if } \sigma \geq 0 \\ \frac{1}{\beta} \ll|\tau|-\mu|\sigma| \gg & \text { if } \sigma<0\end{array}\right.$.

In this criterion, $\sigma$ and $\tau$ are respectively the normal and tangential Cauchy stresses at the integration point where the fracture criterion is evaluated, $\beta=\frac{K_{I C}}{K_{I C}}$ is the coupling mode parameter between mode II and mode I toughness, and $\mu$ is the friction parameter, these two last parameters depending 
Fig. 4 Mode I effective opening $\Delta_{n}^{\star}$.

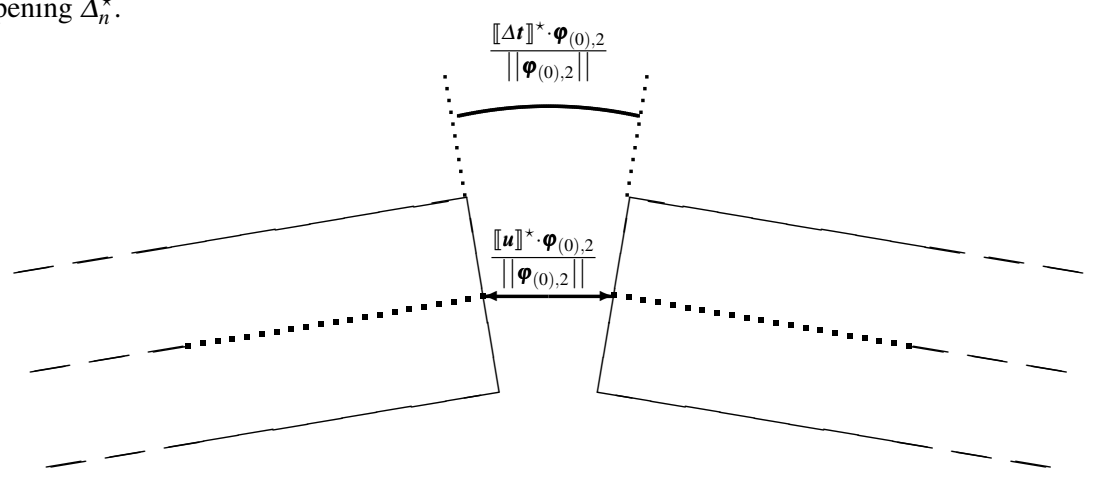

Fig. 5 Mode II effective opening $\Delta_{t}^{\star}$.

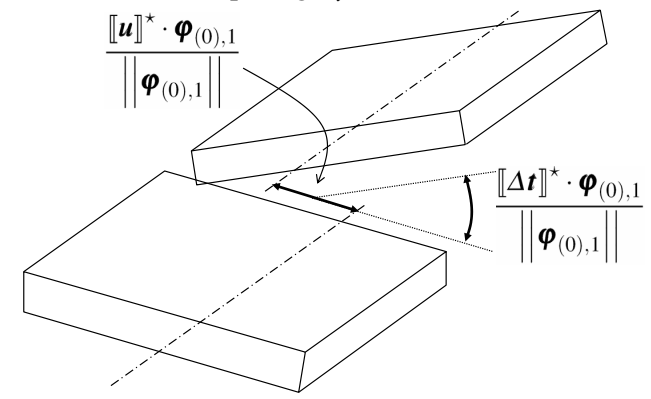

on the material only. The operator $\ll \bullet \gg$ is equal to $\bullet$ if $\bullet \geq 0$ and 0 otherwise. This criterion developed for $3 \mathrm{D}$ cases can still be employed for shells by evaluating the effective stress at the skins of the shell as presented by Becker et al (2011). In fact, the fracture initiates when $\sigma_{\text {eff }}=\sigma_{\mathrm{c}}$ at the shell skin, $\sigma_{\mathrm{c}}$ being the material strength. The determination of $a_{\text {cohesive }}^{s}\left(\llbracket \boldsymbol{\varphi}_{h} \rrbracket, \llbracket \delta \varphi \rrbracket\right)$ requires more
developments. Indeed the implicit discretization of the thickness inherent to shell formulations is not suitable for crack propagating along the thickness, especially in bending, which introduces a compressive stress on a part of the thickness and a tensile stress on the other part. Indeed, as it is brought to light by equation (56) the fracture behavior is different in tension and compression leading to the necessity of moving the neutral axis during fracture propagation, which is very difficult to implement. To avoid this drawback, Becker et al (2011); Becker and Noels (2012) have suggested to apply the cohesive principle directly on the resultant stresses $\boldsymbol{n}$ (14) and $\tilde{\boldsymbol{m}}$ (15). Thus, the equations of the cohesive model used to define $a_{\text {cohesive }}^{s}\left(\llbracket \boldsymbol{\varphi}_{h} \rrbracket, \llbracket \delta \boldsymbol{\varphi} \rrbracket\right)$ can be summarized as follow, with the convention depicted on Figure 3 for the basis vectors definition. It has to be mentioned that the evaluation of the parameters depends on the formulation. On one hand in the linear case, they are evaluated in the reference frame $\boldsymbol{\varphi}_{0, \alpha}$. On the other hand, for the non linear formulation this evaluation is performed in the current configuration $\boldsymbol{\varphi}_{, \alpha}$ to account for the large deformations before crack initiations or propagation. Therefore we unify both cases by using the notation $\boldsymbol{\varphi}_{(0), \alpha}$.

First an effective opening, accounting for the normal and for the tangential jumps is defined. Normal and tangential openings have a contribution coming from the membrane mode of the shell, and a contribution coming from the bending mode:

$$
\begin{aligned}
& \Delta_{n}^{\star}=\left(1-\eta_{I}\right) \frac{\llbracket \boldsymbol{u} \rrbracket^{\star} \cdot \boldsymbol{\varphi}_{(0), 2}}{\left\|\boldsymbol{\varphi}_{(0), 2}\right\|} \pm \eta_{I} h_{I}^{\mathrm{eq}} \frac{\llbracket \Delta \boldsymbol{t} \rrbracket^{\star} \cdot \boldsymbol{\varphi}_{(0), 2}}{\left\|\boldsymbol{\varphi}_{(0), 2}\right\|}, \\
& \Delta_{t}^{\star}=\left(1-\eta_{I I}\right) \frac{\llbracket \boldsymbol{u} \rrbracket^{\star} \cdot \boldsymbol{\varphi}_{(0), 1}}{\left\|\boldsymbol{\varphi}_{(0), 1}\right\|} \pm \eta_{I I} h_{I I}^{\mathrm{eq}} \frac{\llbracket \Delta \boldsymbol{t} \rrbracket^{\star} \cdot \boldsymbol{\varphi}_{(0), 1}}{\left\|\boldsymbol{\varphi}_{(0), 1}\right\|}, \\
& \Delta^{\star}=\sqrt{\ll \Delta_{n}^{\star} \gg^{2}+\beta^{2} \Delta_{t}^{\star 2}}, \\
& h_{I}^{\mathrm{eq}}=\frac{\left|\tilde{m}_{0}^{22}\right|}{h \sigma_{I}-n_{0}^{22}}, \\
& h_{I I}^{\mathrm{eq}}=\frac{\left|\tilde{m}_{0}^{21}\right|}{h \tau_{I I}-n_{0}^{21}}, \\
& \eta_{I}=1-\frac{n_{0}^{22}}{h \sigma_{I}} \text { and, } \\
& \eta_{I I}=1-\frac{n_{0}^{21}}{h \tau_{I I}} \text {. }
\end{aligned}
$$

In these expressions $\Delta_{n}^{\star}$ and $\Delta_{t}^{\star}$ are respectively the effective normal and tangential resultant opening displacements, see Figures 4 and 5 for a physical interpretation. Star terms $\llbracket \boldsymbol{u} \rrbracket^{\star}$ and $\llbracket \Delta \boldsymbol{t} \rrbracket^{\star}$ are respectively the effective displacement and rotation openings resulting from the use of a DG method before fracture activation. Indeed, displacement and rotation jumps are not exactly equal to 0 at fracture initialization due to the weak continuity enforcement. Thus in order to guarantee a null opening at fracture initialization these initial jumps are subtracted from the current displacement and rotational jumps. The equivalent thicknesses $h_{I}^{\mathrm{eq}}$ and $h_{I I}^{\mathrm{eq}}$ are defined to account for the contributions of the rotational opening in 
such a way that the correct amount of energy is released during the crack process. The values of $\sigma_{I}$ and $\tau_{I I}$ are respectively the ones of $\sigma$ and $\tau$ reached in Eq. (56) when $\sigma_{\text {eff }}=\sigma_{\mathrm{c}}$. Finally, ratios $\eta_{I}$ and $\eta_{I I}$ are defined to couple respectively the efforts in tension $n_{0}^{22}$ and in bending $\tilde{m}_{0}^{22}$ responsible for the normal opening and the efforts in shearing $n_{0}^{21}$ and torsion $\tilde{m}_{0}^{21}$ responsible for the tangential opening. The subscript 0 means that these quantities are evaluated at fracture initiation.

Fig. 6 Linear monotonically decreasing cohesive law based on reduced stresses

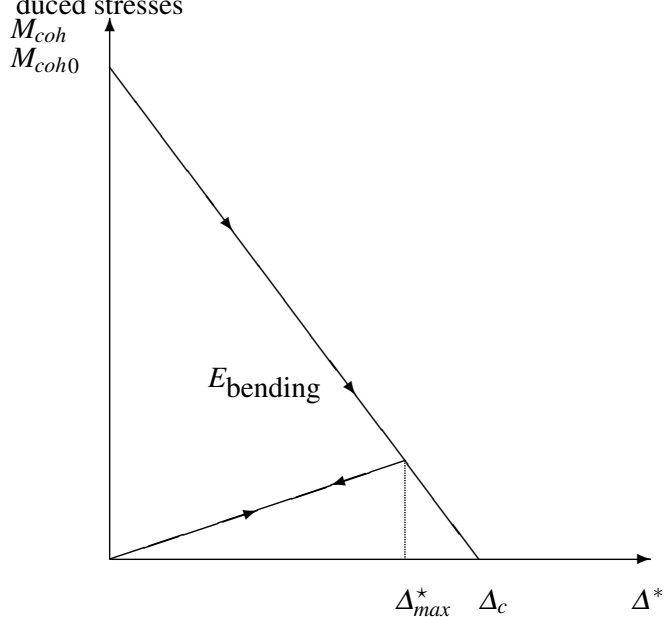

(a) Resultant bending law

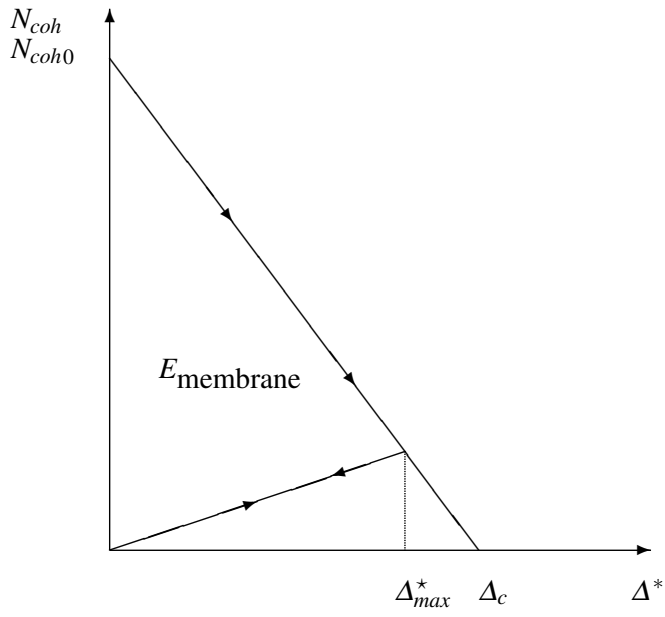

(b) Resultant membrane law

These definitions of the effective openings can now be used to define the contributions to the cohesive law of the membrane and bending openings,

$$
\begin{aligned}
a_{\text {cohesive }}\left(\llbracket \boldsymbol{\varphi}_{h} \rrbracket, \llbracket \delta \boldsymbol{\varphi} \rrbracket\right)= & \int_{s} \bar{j}\left\langle\boldsymbol{n}^{\alpha}\right\rangle_{\mathrm{coh}} \cdot \llbracket \delta \boldsymbol{\varphi} \rrbracket v_{\alpha}^{-} d \partial \mathscr{A}_{e}+ \\
& \int_{s} \bar{j}\left\langle\tilde{\boldsymbol{m}}^{\alpha}\right\rangle_{\mathrm{coh}} \cdot \llbracket \lambda_{h} \delta \boldsymbol{t} \rrbracket v_{\alpha}^{-} d \partial \mathscr{A}_{e},
\end{aligned}
$$

for non-linear applications, while in the linear range, this expression becomes

$$
\begin{aligned}
a_{\text {cohesive }}^{s}\left(\llbracket \boldsymbol{\varphi}_{h} \rrbracket, \llbracket \delta \boldsymbol{\varphi} \rrbracket\right)= & \int_{s} \bar{j}_{0}\left\langle\boldsymbol{n}^{\alpha}\right\rangle_{\text {coh }} \cdot \llbracket \delta \boldsymbol{\varphi} \rrbracket v_{\alpha}^{-} d \partial \mathscr{A}_{e}+ \\
& \int_{s} \bar{j}_{0}\left\langle\tilde{\boldsymbol{m}}^{\alpha}\right\rangle_{\mathrm{coh}} \cdot \llbracket \delta \Delta \boldsymbol{t} \rrbracket v_{\alpha}^{-} d \partial \mathscr{A}_{e},
\end{aligned}
$$

The determination of $\left\langle\boldsymbol{n}^{\alpha}\right\rangle_{\text {coh }}$ and $\left\langle\tilde{\boldsymbol{m}}^{\alpha}\right\rangle_{\text {coh }}$ depends on the cohesive law. As it is well known that for brittle materials the shape of the cohesive law has a little influence on numerical results, as long as the law is monotonically decreasing, a simple linear decreasing law is considered in this work, in which case one can define the critical opening $\Delta_{c}=\frac{2 G_{c}}{\sigma_{c}}$ from the fracture energy $G_{C}$. In case of unloading when $\Delta^{\star}$ becomes lower than the maximal opening reached $\Delta_{\text {max }}^{\star}$, the cohesive forces decrease linearly to zero.

By application of the cohesive principle on the resultant stress vectors, the cohesive model illustrated on Figure 4 is defined as follows:

(i) Tensile case $(\sigma \geq 0 \text { at mid-surface })^{3}$,

(i.a) if $\Delta^{\star} \geq \Delta_{\max }^{\star}$ (loading case),

$$
\begin{aligned}
& \left\langle\tilde{m}^{22}\right\rangle_{\mathrm{coh}}=\tilde{m}_{0}^{22}\left(1-\frac{\Delta^{\star}}{\Delta_{c}}\right) \frac{\Delta_{n}^{\star}}{\Delta^{\star}}, \\
& \left\langle n^{22}\right\rangle_{\mathrm{coh}}=n_{0}^{22}\left(1-\frac{\Delta^{\star}}{\Delta_{c}}\right) \frac{\Delta_{n}^{\star}}{\Delta^{\star}}, \\
& \left\langle\tilde{m}^{21}\right\rangle_{\mathrm{coh}}=\tilde{m}_{0}^{21} \beta\left(1-\frac{\Delta^{\star}}{\Delta_{c}}\right) \frac{\left|\Delta_{t}^{\star}\right|}{\Delta^{\star}}, \\
& \left\langle n^{21}\right\rangle_{\mathrm{coh}}=n_{0}^{21} \beta\left(1-\frac{\Delta^{\star}}{\Delta_{c}}\right) \frac{\left|\Delta_{t}^{\star}\right|}{\Delta^{\star}},
\end{aligned}
$$

(i.b) if $\Delta^{\star}<\Delta_{\max }^{\star}$ (unloading case),

$$
\begin{aligned}
& \left\langle\tilde{m}^{22}\right\rangle_{\mathrm{coh}}=\tilde{m}_{0}^{22}\left(\frac{\Delta^{\star}}{\Delta_{\text {max }}^{\star}}-\frac{\Delta^{\star}}{\Delta_{c}}\right) \frac{\Delta_{n}^{\star}}{\Delta^{\star}}, \\
& \left\langle n^{22}\right\rangle_{\mathrm{coh}}=n_{0}^{22}\left(\frac{\Delta^{\star}}{\Delta_{\text {max }}^{\star}}-\frac{\Delta^{\star}}{\Delta_{c}}\right) \frac{\Delta_{n}^{\star}}{\Delta^{\star}}, \\
& \left\langle\tilde{m}^{21}\right\rangle_{\mathrm{coh}}=\tilde{m}_{0}^{21} \beta\left(\frac{\Delta^{\star}}{\Delta_{\max }^{\star}}-\frac{\Delta^{\star}}{\Delta_{c}}\right) \frac{\left|\Delta_{t}^{\star}\right|}{\Delta^{\star}}, \\
& \left\langle n^{21}\right\rangle_{\mathrm{coh}}=n_{0}^{21} \beta\left(\frac{\Delta^{\star}}{\Delta_{\max }^{\star}}-\frac{\Delta^{\star}}{\Delta_{c}}\right) \frac{\left|\Delta_{t}^{\star}\right|}{\Delta^{\star}} .
\end{aligned}
$$

(ii) Compression case $(\sigma<0$ at mid-surface),

\footnotetext{
${ }^{3}$ Note that the cohesive zone is in terms of the traction components $n^{\alpha \beta}$ and not $\tilde{n}^{\alpha \beta}$
} 
(ii.a) if $\Delta^{\star} \geq \Delta_{\max }^{\star}$ (loading case),

$$
\begin{aligned}
& \left\langle\tilde{m}^{21}\right\rangle_{\mathrm{coh}}=\tilde{m}_{0}^{21}\left(1-\frac{\Delta^{\star}}{\Delta_{c}}\right), \\
& \left\langle n^{21}\right\rangle_{\mathrm{coh}}=n_{0}^{21}\left(1-\frac{\Delta^{\star}}{\Delta_{c}}\right),
\end{aligned}
$$

(ii.b) if $\Delta^{\star}<\Delta_{\max }^{\star}$ (unloading case),

$$
\begin{aligned}
& \left\langle\tilde{m}^{21}\right\rangle_{\mathrm{coh}}=\tilde{m}_{0}^{21}\left(\frac{\Delta^{\star}}{\Delta_{\max }^{\star}}-\frac{\Delta^{\star}}{\Delta_{c}}\right), \\
& \left\langle n^{21}\right\rangle_{\mathrm{coh}}=n_{0}^{21}\left(\frac{\Delta^{\star}}{\Delta_{\max }^{\star}}-\frac{\Delta^{\star}}{\Delta_{c}}\right),
\end{aligned}
$$

where in this last case the condition $\frac{\left|\Delta_{t}^{\star}\right|}{\Delta^{\star}}=\frac{1}{\beta}(c f$. Eq. (59)) is taken into account.

The use of $n_{0}^{22}, \tilde{m}_{0}^{22}, n_{0}^{21}, \tilde{m}_{0}^{21}$ allows guarantying the continuity of interface forces at fracture initialization. If this continuity is not ensured, Papoulia et al (2003) have demonstrated that there are some convergence problems. Furthermore, as at fracture initialization the ratios $\frac{\Delta_{n}^{\star}}{\Delta^{\star}}$ and $\frac{\mid \Delta_{t}^{\star}}{\Delta^{\star}}$ are undetermined, their initial values are chosen equal to respectively 1 and $\frac{1}{\beta}$ in order to ensure the continuity of efforts. Note that the choice of the tensile or compressive case is performed at fracture initialization.

The choice of such a linearly decreasing cohesive law is motivated by the applications considered in this paper, which are either with brittle materials or elasto-plastic materials under the small-scale yielding assumption. However, when considering ductile fracture, mainly for metals, the stress triaxiality modifies the critical strains. The stress triaxiality is not the only indicator that should be accounted for, as axisymmetric and shear-dominated stress states imply different fracture strains as shown by Barsoum and Faleskog (2007); Gao and Kim (2006); Nahshon and Hutchinson (2008). Recently, damage models have been developed or improved to account for these behaviors, see Nahshon and Hutchinson (2008); Nielsen and Tvergaard (2009); Scheyvaerts et al (2011) among others. An advantage of the DG/ECL framework proposed is its ability to integrate damage models at the interface, and thus to give more physical insight to fracture criterion and to the cohesive law. Another possibility is to calibrate the cohesive law to account for the particular plane-stress state. The determination of cohesive energy associated to plane-stress crack growths in ductile materials can account for the energy dissipated during necking, shear localization and slant fracture following the onset of necking as pointed out by Scheider and Brocks (2006); Nielsen and Hutchinson (2011). Finally, when considering shell elements, the implicit representation of the thickness usually restricts fracture mechanics to through-the-thickness cracks normal to the mid-surface and limits the 3D effects of the crack that can be accounted for. Although the cohesive model presented in this section allows considering partially fractured thickness, more physical insight could be obtained
Fig. 8 Crack propagation for the 3-point bending plate. Results are in agreement with Zavattieri (2006).

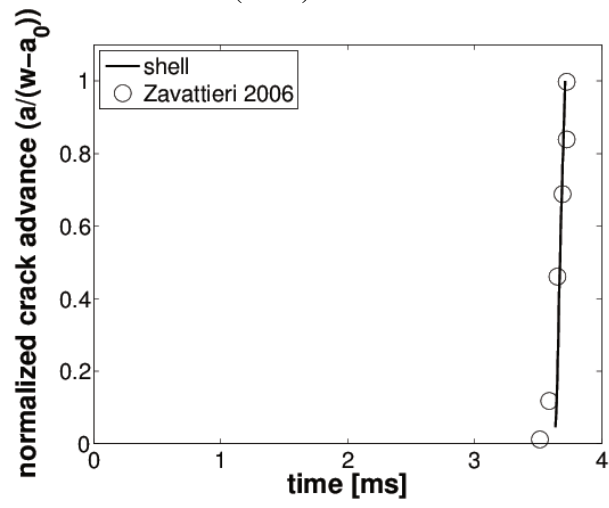

Table 1 Material properties for the 3-point bending plate.

\begin{tabular}{ll}
\hline Property & Value \\
\hline Young modulus [GPa] & 200 \\
Poisson ratio [-] & 0.3 \\
Density $\left[\mathrm{kg} / \mathrm{m}^{3}\right]$ & 7850 \\
Fracture energy $\left[\mathrm{J} / \mathrm{m}^{2}\right]$ & 24500 \\
Fracture strength $[\mathrm{MPa}]$ & 2170 \\
Coupling parameter $\beta[-]$ & 1 \\
Frictional coefficient $[-]$ & 0. \\
\hline
\end{tabular}

by considering locally $3 \mathrm{D}$ elements as suggested by Wyart et al (2007).

\section{Applications}

The ability of the DG/ECL framework presented in this paper to model different fracture phenomena is demonstrated in this section through 4 numerical benchmarks. The two first ones investigate the crack propagation in an elastic plate and in an elasto-plastic cylinder, both having an initial notch. The last two examples study the fragmentation of a ring and of a sphere made of a brittle material using the linearelasticity assumption.

\subsection{Three-point bending plate}

This benchmark focuses on a simply supported notched plate dynamically impacted in its center by a rigid cylinder of $0.01[\mathrm{~m}]$-diameter, with a prescribed velocity of $1[\mathrm{~m} / \mathrm{s}]$. The setup of this benchmark, including the dimensions, is depicted on Figure 8(a) and the material values are reported in Table 1. The crack propagation was previously reported in the literature by Zavattieri (2006), who used shell elements combined with an intrinsic cohesive law for which cohesive elements are pre-inserted along the crack path. Furthermore 
Fig. 7 Setup of the 3-point bending plate as previously presented by Zavattieri (2006).

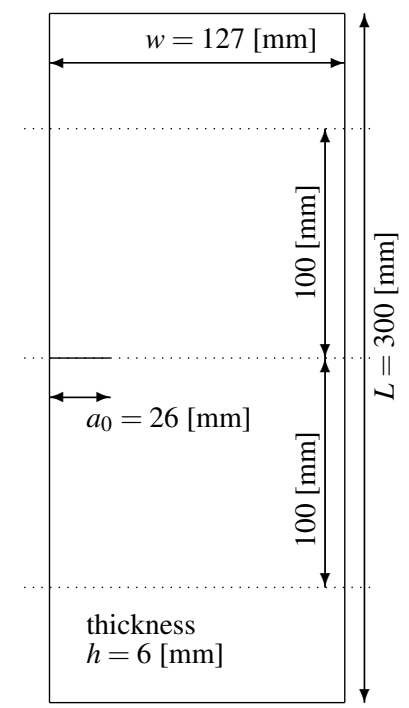

(a) Dimensions of the plate. The dotted lines represent the axes of the cylinders

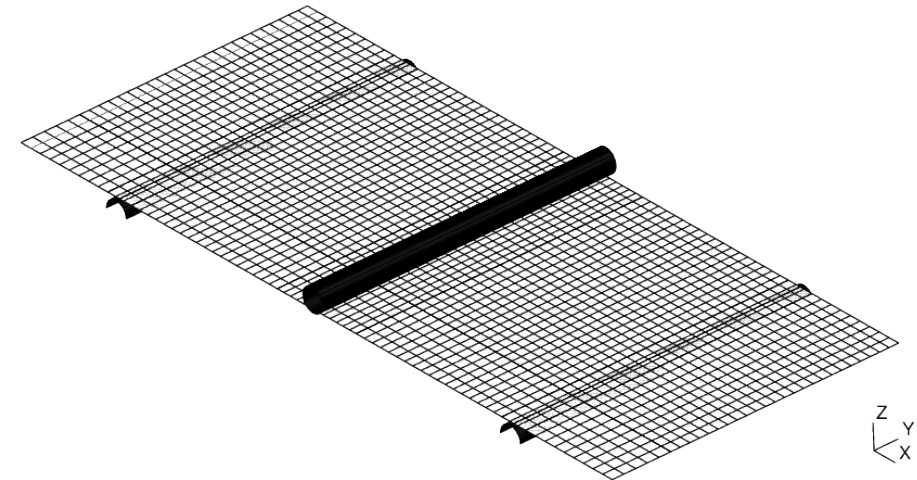

(b) Mesh he used a fracture criterion based on a maximal bending momentum, which is transformed herein to a criterion in $\sigma_{\mathrm{c}}$. The mesh used, Figure $8(\mathrm{~b})$, has $40 \times 80$ cubic quadrangle elements. Finally, although the crack path is well defined for this case, due to the loading conditions, in our framework crack can propagate along any interface elements. This shows that the stress wave propagation is not modified by the DG/ECL framework.

The simulation is performed on 4 CPUs using Hulbert and Chung (1996) time-integration algorithm, and without numerical dissipation. The stability parameters chosen accordingly to Becker et al (2011) are $\beta_{1}=\beta_{2}=10$., $\beta_{3}=$ 0.0001 . The crack propagation over time is displayed on Figure 8 which also shows the results obtained by Zavattieri (2006). The crack propagation initiates at the same time and the plate is fully broken at the same time too.

\subsection{Blasted notched-cylinder}

This benchmark focuses on a axially notched cylinder dynamically loaded by a blast wave. In particular the speed of the crack propagation is studied. The use of a linear material law leads to a spurious high speed of propagation, as shown by Larsson et al (2011), see Figure 10(b), and therefore the non-linear formulation (40) is used in combination with a $J_{2}$-flow material law (Section 2.4.2). The cylinder has a diameter of $1.2[\mathrm{~m}]$, is $1[\mathrm{~m}]$ long and has a thickness of
Table 2 Material properties for the blasted cylinder.

\begin{tabular}{ll}
\hline Properties & Values \\
\hline Young modulus [GPa] & 73.1 \\
Poisson ratio $[-]$ & 0.33 \\
Density $\left[\mathrm{kg} / \mathrm{m}^{3}\right]$ & 2780 \\
Yield stress $[\mathrm{MPa}]$ & 350 \\
Hardening modulus [MPa] & 800 \\
Fracture energy $\left[\mathrm{J} / \mathrm{m}^{2}\right]$ & 19000 \\
Nominal fracture strength $[\mathrm{MPa}]$ & 650 \\
Coupling parameter $\beta[-]$ & 1 \\
Frictional coefficient $[-]$ & 0. \\
\hline
\end{tabular}

1 [mm]. It exhibits an initial crack of 56 [mm] centered on its height. The material is the Al2024-T3, with the properties reported in Table 2. In order to avoid unphysical blow up of elements during crack propagation, the idea suggested by Zhou and Molinari (2004b), who used statistical distributions for the fracture strength $\sigma_{c}$, is considered. This strength can vary in a range around its nominal value $(10 \%$ for the presented application) at each Gauss point of the interface elements, which is physically justified by the material imperfections.

Due to the symmetry and to save computational time, only the top side of the cylinder is modeled with an unstructured mesh of 18536 cubic triangles, see Figure 10(c). The simulation is performed with the Hulbert and Chung (1996) 
Fig. 9 Model and results for the blasted cylinder.

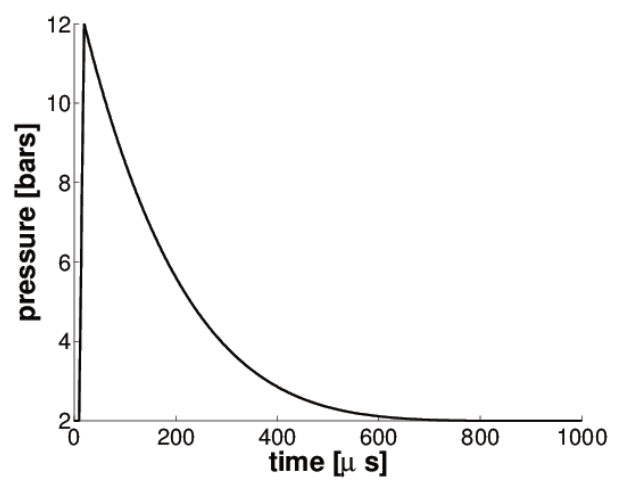

(a) Applied pressure

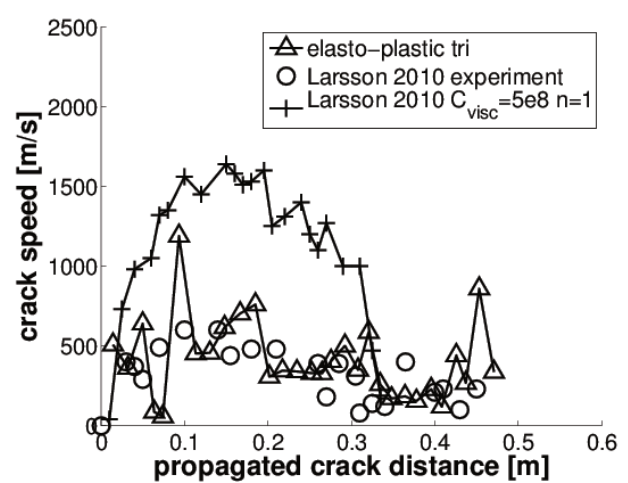

(b) Crack speed

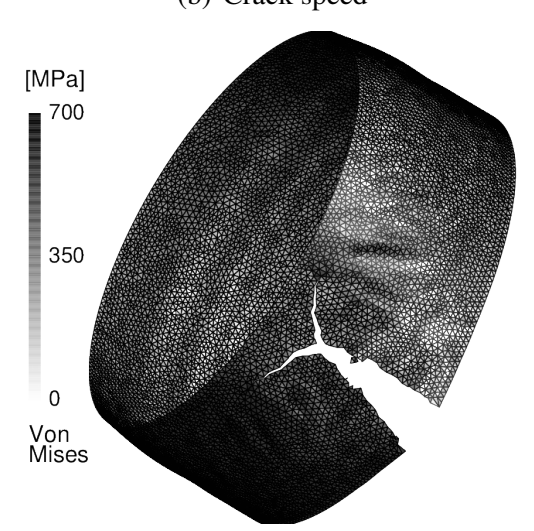

(c) Cylinder at the end of simulation

explicit time integration scheme including a low numerical dissipation (spectral radius of 0.9). The blast is simulated thanks to the curve depicted on Figure 10(a). The speed of crack propagation is studied on Figure 10(b), which shows the crack evolution obtained during the experiments, for the simulations achieved by Larsson et al (2011), and also for the presented DG/ECL framework. As predict by Larsson et $a l$. the introduction of plasticity allows obtaining results in agreement with experiments even if the crack speed at the beginning seems faster in our model. After a propagation of $0.18[\mathrm{~m}]$ the model matches quite well the experimental data, see Figure 10(c).

\subsection{Fragmentation problems}

The previously presented results demonstrate the ability of the full-discontinuous Galerkin / extrinsic cohesive law framework to propagate an initial crack. Hereafter, the authors investigate the ability of such a model to initiate cracks. An interesting case of multiple crack initiations is the case of fragmentation. Recently, several authors developed the framework presented herein for 3D elements (in place of shell elements). On the one hand, Radovitzky et al (2011) studied the fragmentation of a thick plate due to the impact of a rigid sphere. On the other hand Levy (2010) applied the same framework to the uniform expansion of a hollow sphere. This last case, can be solved with the shell linear formulation (54) when the thickness is small enough (the thickness of the sphere is $0.1[\mathrm{~mm}]$ for an external radius of $10[\mathrm{~mm}])$, which allows to model the sphere as a thin body. Furthermore, the literature reports other fragmentation studies as, among others, the one presented by Zhou and Molinari (2004a). In this reference the fragmentation of a plate ring under radial uniform expansion is studied with classical extrinsic cohesive law for 3D elements. As they used a continuous formulation only serial computations were performed with a high computational time. The DG/ECL framework presented in this paper allows using a parallel implementation and leads to a reduced computational time.

In the following, through the study of two benchmarks, a successful comparison between the $3 \mathrm{D}$ formulations of the literature and the shell framework presented herein is carried on.

\subsubsection{Defect model: Weibull distribution}

The mechanism of fragmentation is mainly controlled by the distribution of the defects in the specimen. Indeed, for a specimen made of a perfect material and uniformly loaded the fracture takes place in each point at the same time. Thus, the number of fragments obtained by a finite element analysis is equal to the number of elements contained in the mesh. On the contrary, in a component made of a non perfect material the fracture initiations of multiple cracks take place near the defects, as these are the location of stress concentration in the microstructure. Obviously, fracture occurs earlier at these stress concentrations and forms fragments composed of several elements. The model of these defects is not straightforward in a finite element analysis as they cannot be represented by simple parameters. So a statistical distribution of the cohesive strength is commonly used as suggested by Leterrier et al (1997); Zhou and Molinari (2004a); Dickens and Cho (2005); Levy (2010). 
Although to model the fragmentation, the cohesive model presented in Section 4 could be used, in this section we consider a more elaborate statistical distribution of the strength $\sigma_{\mathrm{c}}$ to be consistent with the fragmentation data of the literature. The most employed statistical model for defects distributions, and used hereafter, is based on the work of Weibull (1939). He performed some experiments which demonstrated that the tensile loading leading to fracture can vary for specimens of the same material and of the same geometry. Based on these experiments he suggested an empirical formula for the probability of fracture, which accounts for the heterogeneity of the material,

$P_{f}(\sigma, V)=1-\exp ^{-N(\sigma, V)}$,

where $\sigma$ is the value of the equivalent stress and where $V$ is the volume of the specimen. The function $N(\sigma, V)$ increases necessarily monotonically with $\sigma$ and, therefore, Weibull (1939) suggested, based on his experimental data, to employ a power law,

$N(\sigma, V)= \begin{cases}\frac{V}{V_{0}}\left(\frac{\sigma-\sigma_{\min }}{\sigma_{0}}\right)^{m} & \text { if } \sigma \geq \sigma_{\min } \\ 0 & \text { otherwise }\end{cases}$

where $V_{0}$ is a arbitrary normalizing volume. Furthermore the other parameters $\sigma_{0}, \sigma_{\min }$ and $m$, representing respectively a stress scale factor, the minimal value for which the fracture can occur and the Weibull modulus, are all characteristics of the material.

The work of Weibull can be applied on the cohesive strength, which is then computed from

$F\left(\sigma_{\mathrm{c}}\right)=1-\exp ^{-\left(\frac{\sigma_{\mathrm{c}}-\sigma_{c, \min }}{\sigma_{0}}\right)^{m}}$.

In practice it is easier to generate a uniform distribution between 0 and 1 , so a Weibull distribution can be easily obtained from

$\sigma_{\mathrm{c} s}=\sigma_{0}(-\log (\text { xrand }))^{\frac{1}{m}}+\sigma_{c, \text { min }}$,

with $\sigma_{\mathrm{c} s}$ the value of the cohesive strength for the interface $s$ and $x$ rand a random value coming from a uniform distribution between 0 and 1 .

\subsubsection{Fragmentation of a plate ring}

The first example of fragmentation presented herein focuses on a thin plate ring under radial expansion, as shown on Figure 10 and with the material properties given in Table 3. The fragmentation is consequent to a centrifugal force that is simulated, as suggested by Zhou and Molinari (2004a), by prescribing on each mass point $i$ a body force computed as,

$\mathbf{f}_{i}(\mathbf{r})=m_{i} w^{2} \mathbf{r}$,

where $m_{i}$ is the nodal mass, $\mathbf{r}$ is the radial vector of node $i$ and where $w$ is the angular velocity given by,

$w=\left\{\begin{array}{ll}\frac{w_{0} t}{2 \pi t_{0}} & \text { if } t \leq t_{0} \\ \frac{w_{0}}{2 \pi} & \text { if } t>t_{0}\end{array}\right.$.
Fig. 10 Geometry of the plate ring fragmentation.

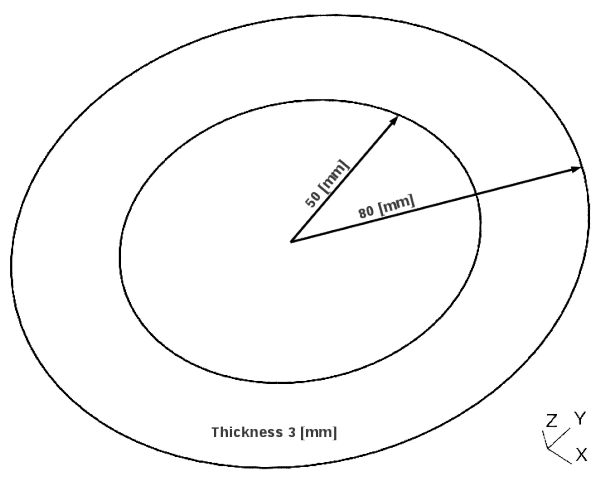

Fig. 11 Weibull distributions: the more a material is homogeneous, the more the Weibull modulus is high and the more the pdf is concentrated around the peak value.

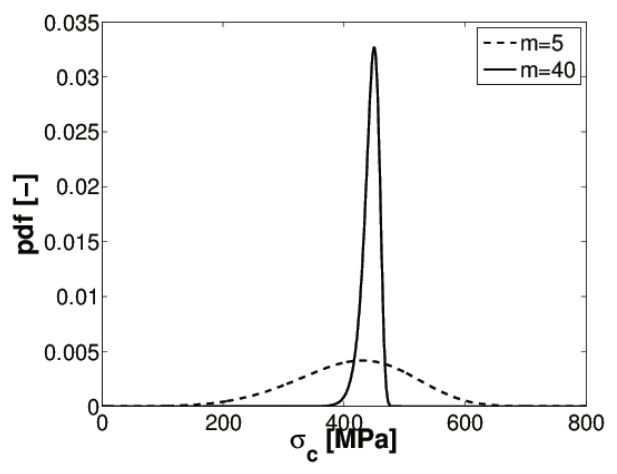

Table 3 Material properties of the plate ring fragmentation.

\begin{tabular}{ll}
\hline Properties & Values \\
\hline Young modulus [GPa] & 320 \\
Poisson ratio [-] & 0.3 \\
Density $\left[\mathrm{kg} / \mathrm{m}^{3}\right]$ & 3300 \\
Fracture energy [J/m $\left.{ }^{2}\right]$ & 200 \\
Minimal fracture strength [MPa] & 0 \\
Weibull modulus [-] & 5 or 40 \\
Fracture strength scale factor [MPa] & 450 \\
Coupling parameter $\beta[-]$ & 1 \\
Frictional coefficient [-] & 0. \\
\hline
\end{tabular}

Values $w_{0}=60000[\mathrm{rps}]$ and $t_{0}=75[\mu \mathrm{s}]$ are considered for the presented simulations.

The ring is meshed with 32380 quadratic triangles to obtain the same number of interfaces than Zhou and Molinari (2004a). The simulation is performed on 16 CPUs for different Weibull moduli (5 and 40) with the explicit Hulbert and Chung (1996) time-integration algorithm associated to low numerical dissipation (spectral radius $=0.95$ ). The stability parameters used are $\beta_{1}=\beta_{2}=10$. In this case, as the out of plane displacement is prescribed, the value of $\beta_{3}$ has no in- 
Fig. 12 Fragmentation process of the plate ring with a Weibull modulus $m=5$. Results are in agreement with Zhou and Molinari (2004a)

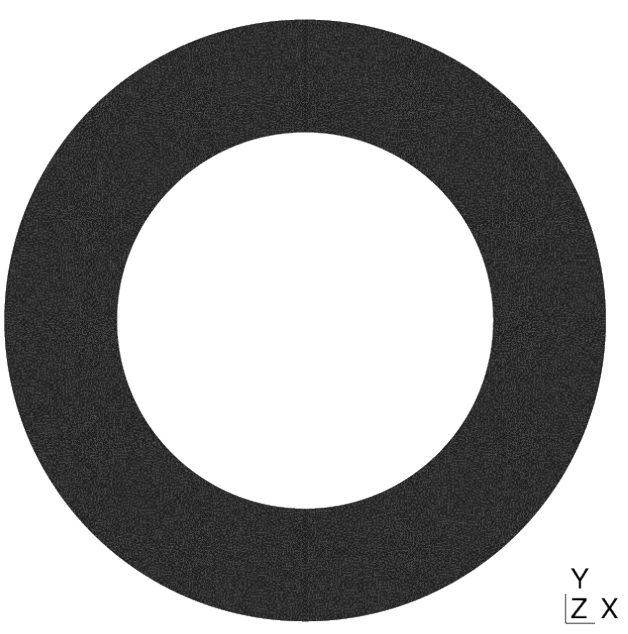

(a) $\mathrm{t}=26[\mu \mathrm{s}]$

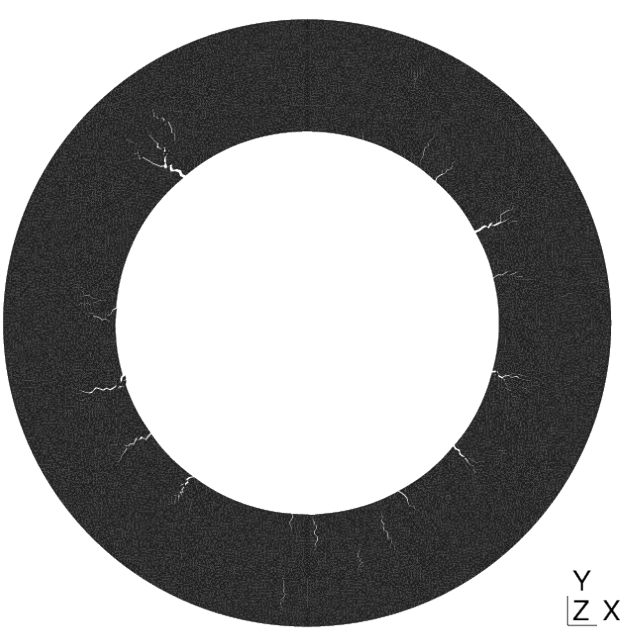

(c) $\mathrm{t}=30[\mu \mathrm{s}]$

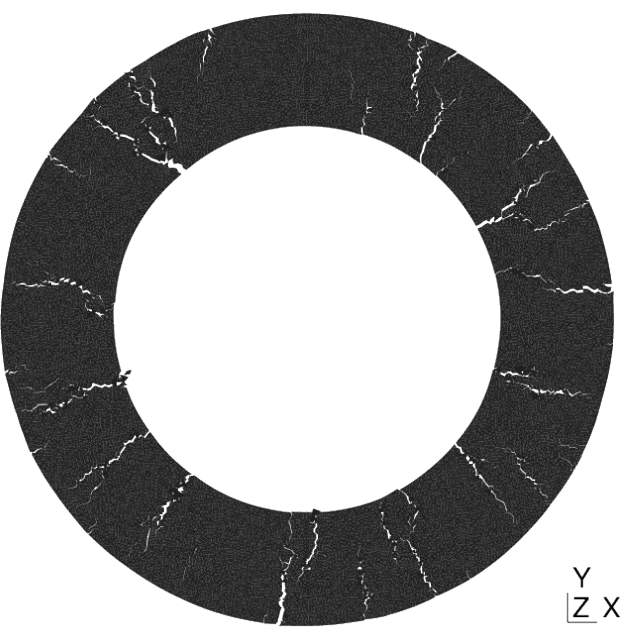

(e) $\mathrm{t}=34[\mu \mathrm{s}]$

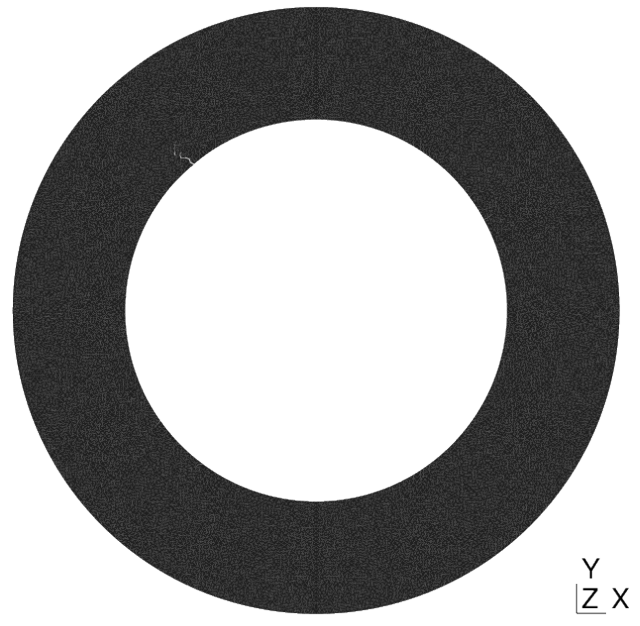

(b) $\mathrm{t}=28[\mu \mathrm{s}]$

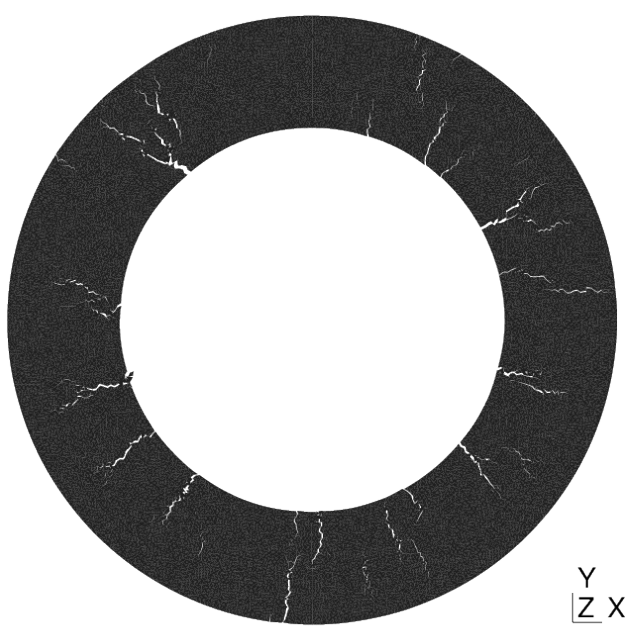

(d) $\mathrm{t}=32[\mu \mathrm{s}]$

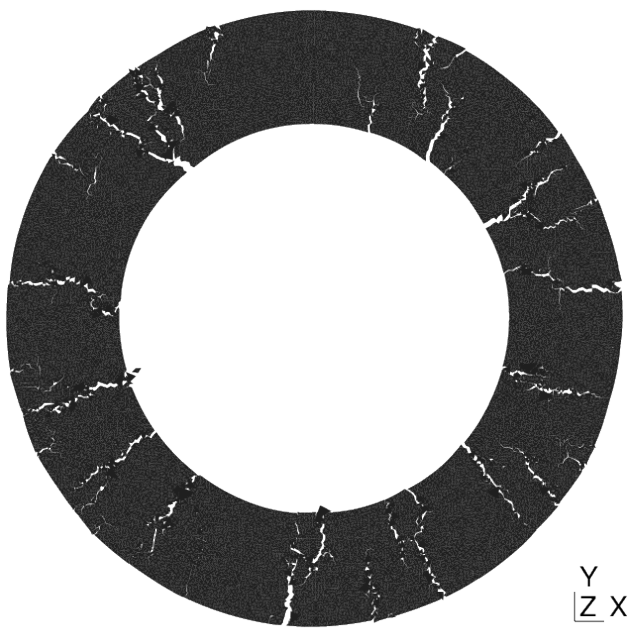

(f) $\mathrm{t}=36[\mu \mathrm{s}]$ 
Fig. 13 Fragmentation process of the plate ring with a Weibull modulus $m=40$. Results are in agreement with Zhou and Molinari (2004a)

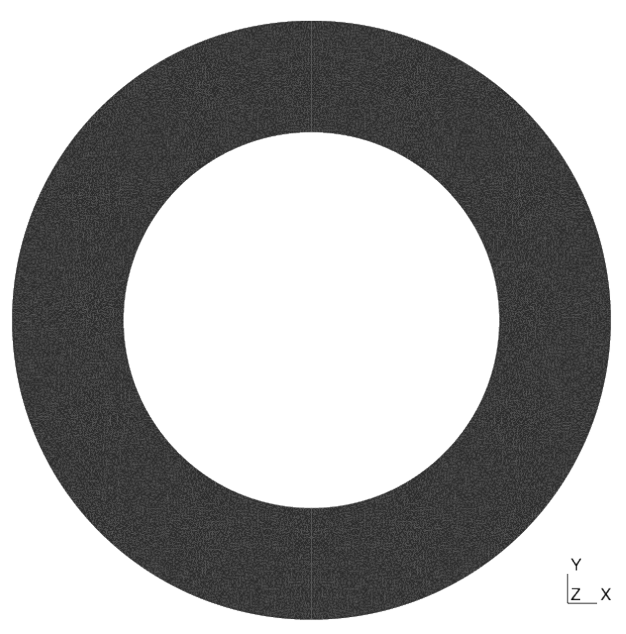

(a) $\mathrm{t}=36[\mu \mathrm{s}]$

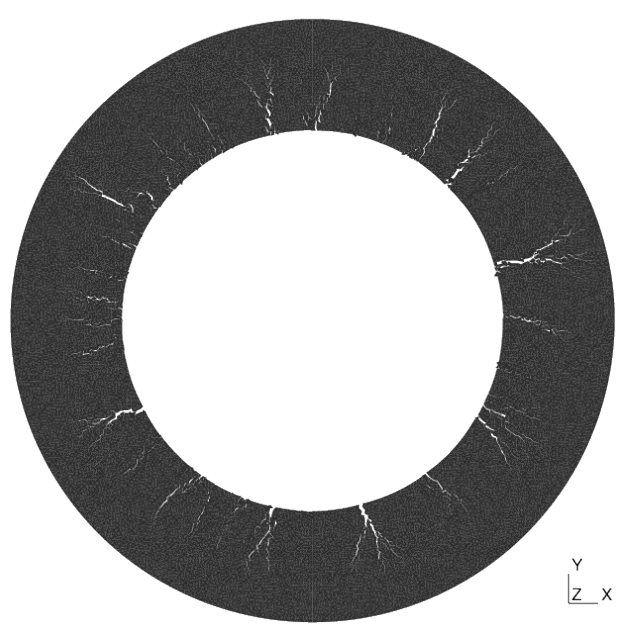

(c) $\mathrm{t}=40[\mu \mathrm{s}]$

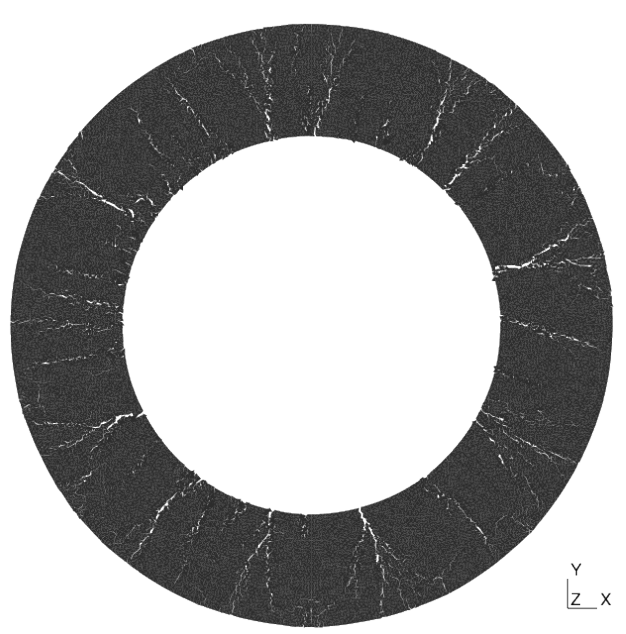

(e) $\mathrm{t}=44[\mu \mathrm{s}]$

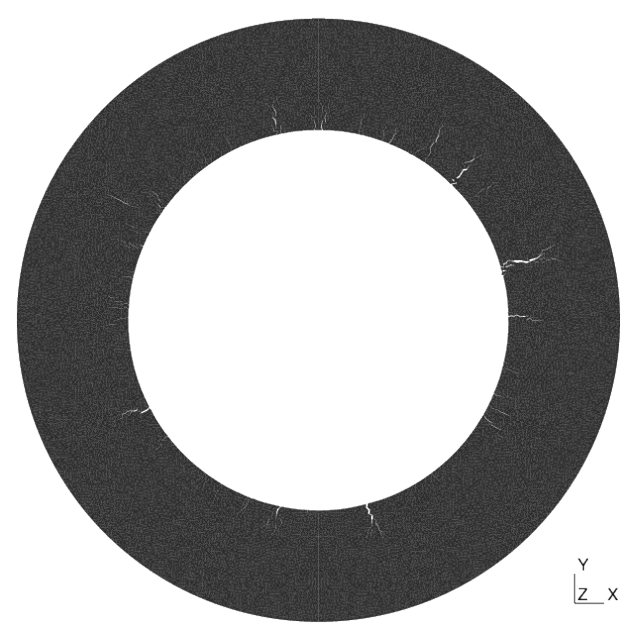

(b) $\mathrm{t}=38[\mu \mathrm{s}]$

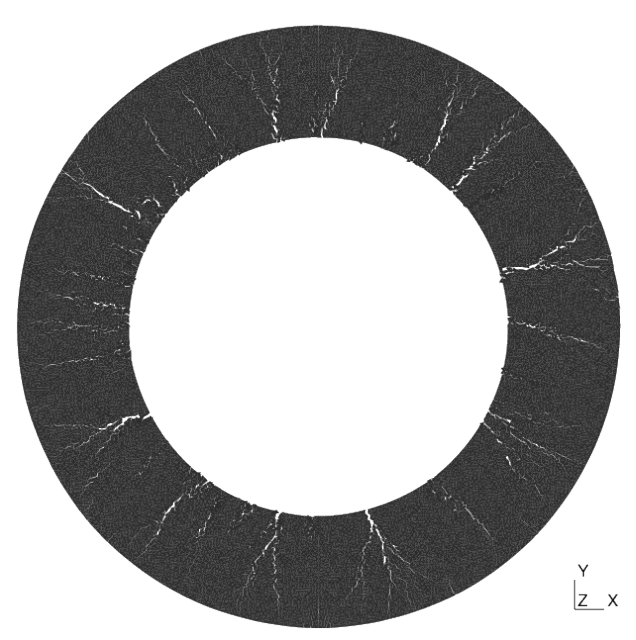

(d) $\mathrm{t}=42[\mu \mathrm{s}]$

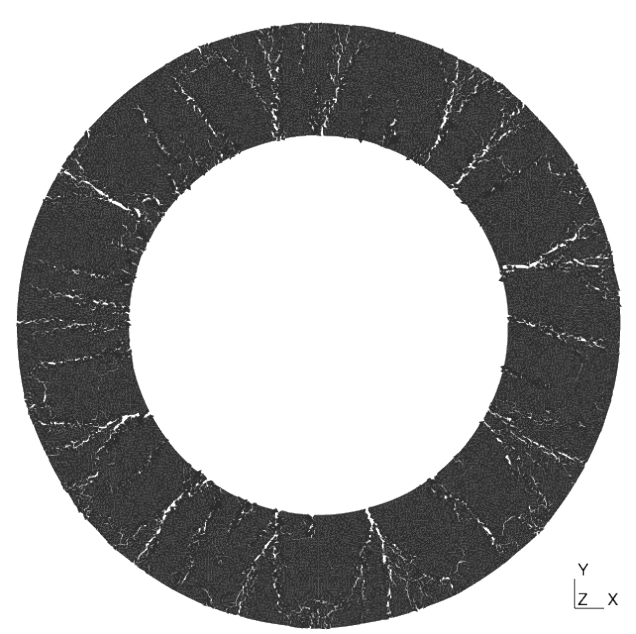

(f) $\mathrm{t}=46[\mu \mathrm{s}]$ 
fluence on the results. Two Weibull moduli are successively considered, which give the strength distributions reported in Figure 11.

For both Weibull moduli, the fragmentation processes, displayed in the Figures 12 for $m=5$ and 13 for $m=40$, are coherent with the results provided by Zhou and Molinari (2004a). For $m=5$, cracks initiate at the inner radius at approximately $28[\mu \mathrm{s}]$ and propagate more or less radially (with crack branching) toward the outer radius. With a Weibull modulus of 40, more cracks (i.e. smaller fragments) are generated and they appear later than for $m=5$. This observation is in agreement with Zhou and Molinari (2004a) who noticed that as "a high Weibull modulus implies a fairly homogeneous material, it is not surprising that under suffcient loading conditions, crack initiation occurs at more locations [...] the ring rotation speed at crack initiation should be an increasing function of the Weibull modulus". However in our simulations, for $m=40$ the cracks reach the outer side after $44[\mu \mathrm{s}]$ instead of the $36[\mu \mathrm{s}]$ reported by Zhou and Molinari (2004a).

\subsubsection{Fragmentation of a sphere}

Table 4 Material properties for the fragmentation of a sphere.

\begin{tabular}{ll}
\hline Properties & Values \\
\hline Young modulus [GPa] & 370 \\
Poisson ratio [-] & 0.22 \\
Density $\left[\mathrm{kg} / \mathrm{m}^{3}\right]$ & 3900 \\
Fracture energy [J/m $\left.{ }^{2}\right]$ & 50 \\
Minimal fracture strength [MPa] & 264 \\
Weibull modulus [-] & 2 \\
Fracture strength scale factor [MPa] & 50 \\
Coupling parameter $\beta[-]$ & 1 \\
Frictional coefficient [-] & 0. \\
\hline
\end{tabular}

The second fragmentation test considers the thin sphere under uniform expansion problem previously analyzed by Levy (2010) with 3D elements. In this work, this simulation is performed with the linear full-discontinuous Galerkin Kirchhoff-Love shell formulation presented in the Section 3. The fracture initiation is modeled by cohesive interface elements as described in Section 4. The cohesive strength of these elements follows a Weibull distribution, see Section 5.3.1. The material parameters of the ceramic sphere are reported in Table 4 . The symmetry of the sphere is taken into account and only $1 / 8^{\text {th }}$ of the sphere is meshed with 144,528 quadratic triangles. As the mesh corresponds to the mid-plane of the sphere, its radius is equal to 9.95 [mm] for a thickness of $0.1[\mathrm{~mm}]$. Note that the very thin mesh used to discretize the sphere leads to consider a problem with approximately 2.6 million of unknowns. This number
Fig. 14 Final configuration of the fragmentation of a sphere with a strain rate of $1 e^{4} \mathrm{~s}^{-1}$.

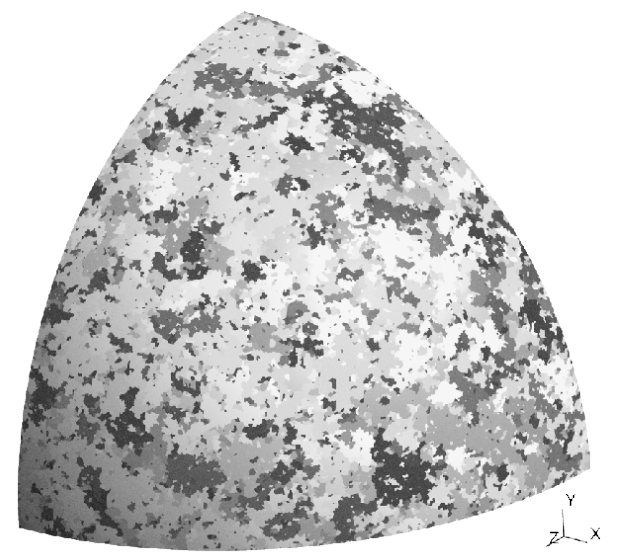

of unknowns is larger than the number used by Levy (2010), which is 818,928 dofs, modeling the sphere with one tetrahedral element over the thickness. The ultra fine meshes was required in our simulation to increase the number of elements per fragment. Nevertheless, this large number of degrees of freedom can be treated by the parallel implementation suggested by Becker and Noels (2012), and an acceptable computational time is obtained using 32 CPUs. The stability parameters are set, following Becker and Noels (2012), to 10,10 and 0.0001 respectively for $\beta_{1}, \beta_{2}$ and $\beta_{3}$. The uniform expansion of the sphere (centered in $(0,0,0)$ ) is simulated by prescribing an initial velocity profile following,

$v_{x}(x, y, z)=\dot{\varepsilon} x$,

$v_{y}(x, y, z)=\dot{\varepsilon} y$, and,

$v_{z}(x, y, z)=\dot{\varepsilon} z$,

where $\dot{\varepsilon}$ is the strain rate. Three different strain rates are successively considered: $1 e^{4}, 2 e^{4}$ and $1 e^{5} \mathrm{~s}^{-1}$ and the final configuration of the sphere is displayed on Figure 14 in the case of the lowest strain rate $\left(\dot{\varepsilon}=1 e^{4} \mathrm{~s}^{-1}\right)$.

Figure 16(a) represents the time evolutions of the kinetic and potential energies of the sphere loaded with a strain rate of $1 e^{5} \mathrm{~s}^{-1}$. As an initial velocity is prescribed the kinetic energy is initially different from zero and decreases with the increase of the potential energy. This one reaches a peak value at time $t_{\text {peak }}$ when part of the potential energy is released during the fracture process which occurs at that time. Afterward, the kinetic and potential energies stabilize. The final time of our simulation is chosen as twice the value of $t_{\text {peak }}$, which differs for the different strain rates. Figure 16(b) shows the energy dissipated by the fracture process which starts to increase when the potential energy begins to decrease as expected. Then it remains constant for a short time before a second increase follows the second peak in the potential energy. At this end of the simulation the dissipated 
Fig. 15 The energy is conserved over time for the fragmentation of a sphere. Illustration with a strain rate of $1 e^{5} \mathrm{~s}^{-1}$.

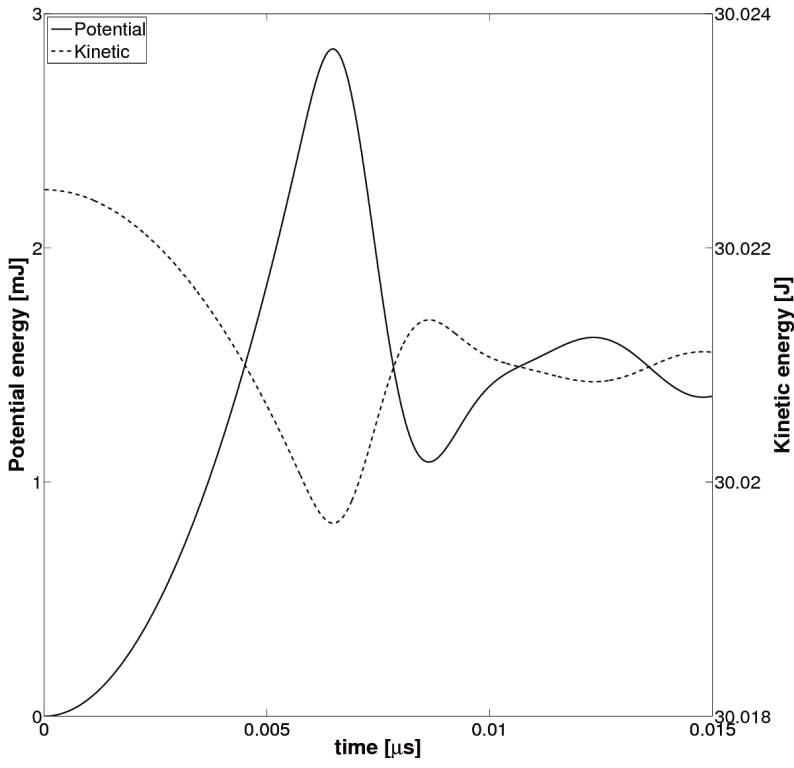

(a) Kinetic and potential energy

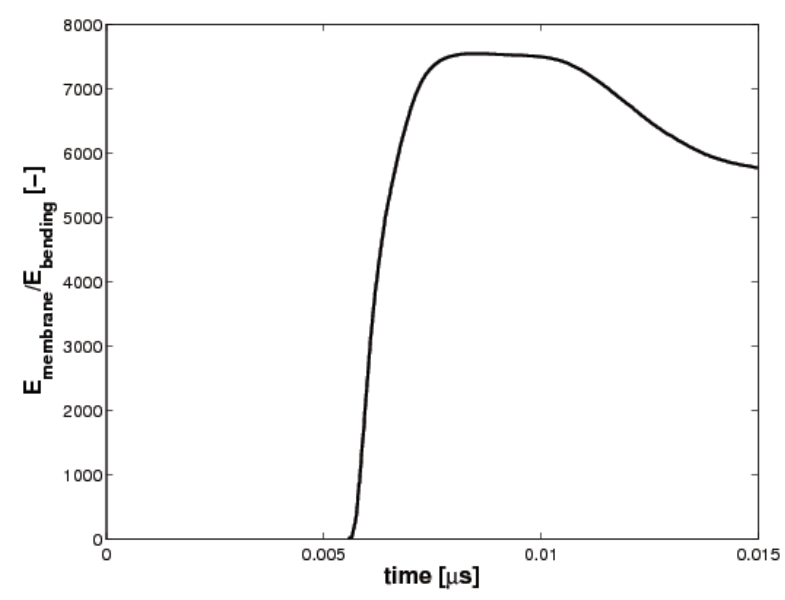

(c) Membrane - bending ratio

energy seems stabilized. Figure 16(c) displays the ratio between the energy dissipated in the membrane mode and the energy released in the bending mode. During the first release of the fracture energy, the major contribution comes from the membrane mode and the energy dissipated by the bending mode is anecdotal. However, during the second release of the fracture energy, a larger bending contribution is involved, as shown by the decrease of the ratio at that time. Finally, the Figure 16(d) proves that our method preserves the energetic balance. Indeed, the variation of the total energy over the whole simulation is about $0.0001 \%$. This low dissipation results from the choice of a spectral radius of 0.95 in the Hulbert-Chung algorithm.

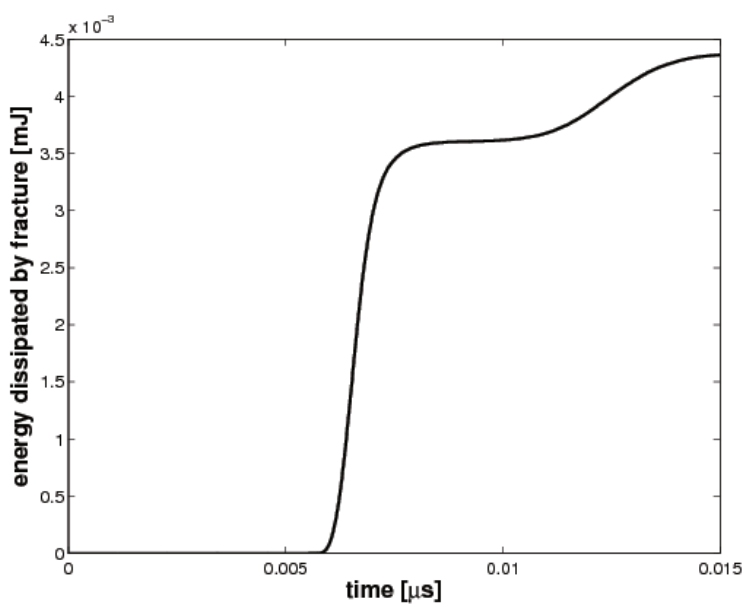

(b) Fracture energy

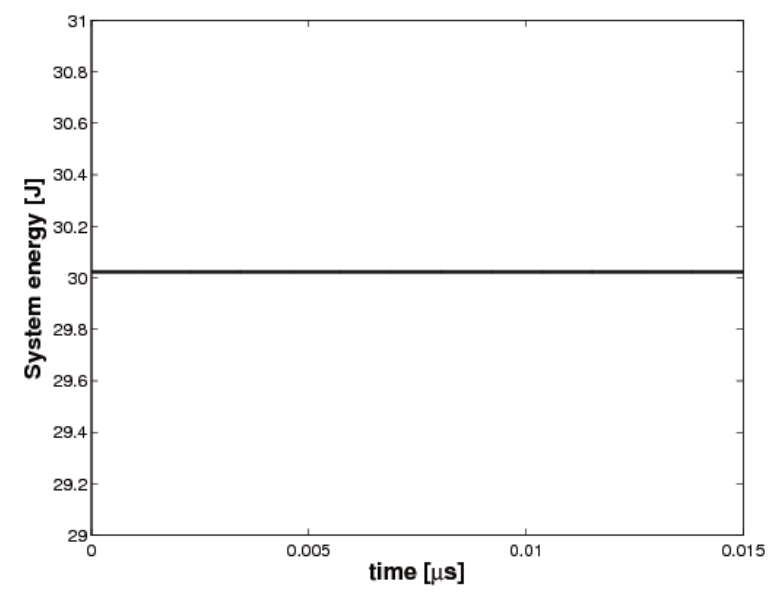

(d) Total energy

Figure 16 represents the mass distribution and the number of fragments obtained in terms of the loading strain rate $\dot{\varepsilon}$. In the presented results, the dust-like fragments (i.e. the fragments composed of only 1 or 2 elements) are neglected as suggested by Levy (2010). The pictures show that the results obtained by the framework presented in this paper are in agreement with the mass distribution and the number of fragments predicted by the work of Levy (2010), at the exception of the largest strain rate for which the shell formulation predicts less fragments. This difference is due to the small number of elements per fragment obtained with our mesh. Indeed, the mean value is around 7 elements by fragment, which may cause a mesh dependency. Overall, our 
Fig. 16 Results for the fragmentation of a sphere with different strain rates. Results obtained with the DG/ECL shell framework are compared to 3D fragmentation results obtained by Levy (2010).

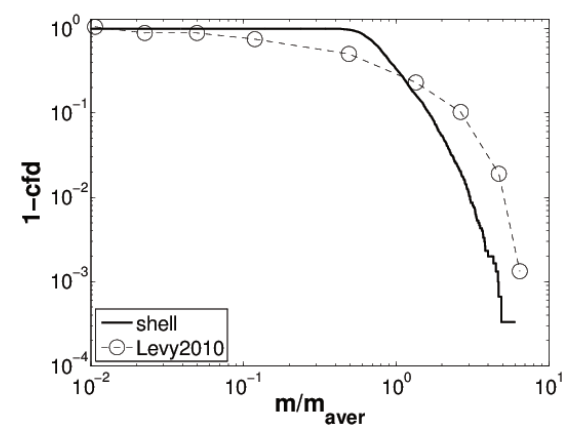

(a) Mass distribution of fragment $\dot{\varepsilon}=1 e 4$

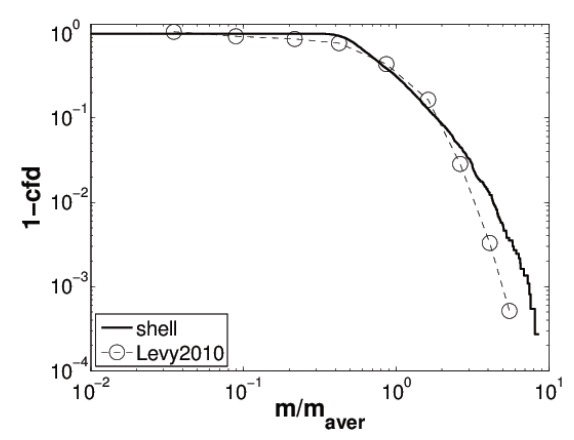

(b) Mass distribution of fragment $\dot{\varepsilon}=2 e 4$

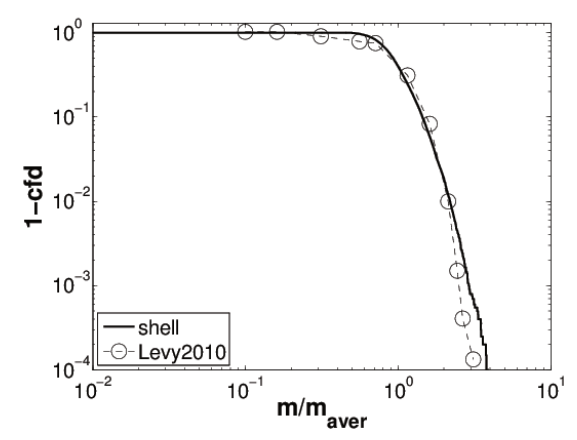

(c) Mass distribution of fragment $\dot{\varepsilon}=1 e 5$

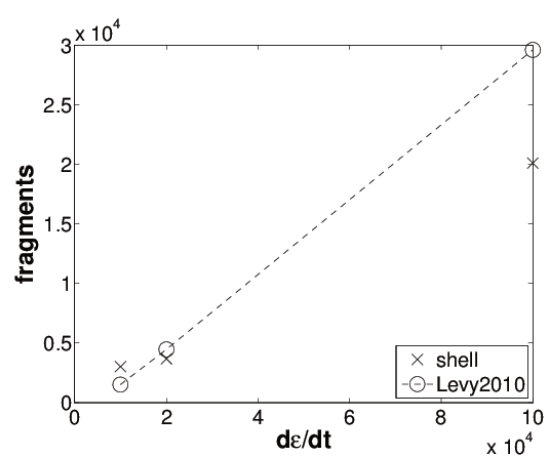

(d) Number of fragments

shell formulation is shown to be an efficient tool to predict fragmentation of brittle materials.

\section{Conclusions}

This paper focuses on the applicability of the DG/ECL framework for shells to model various fracture cases. On the one hand, the recourse to a discontinuous formulation allows inserting cohesive elements on the fly during the simulation without any modification of the mesh, which is an issue with the continuous approach, and on the other hand, it allows obtaining an easy parallel implementation of the resolution scheme. The recourse to a parallel algorithm is mandatory as the number of degrees of freedoms required to capture the fracture process is particularly important.

The presented benchmarks involve very different fracture processes: crack propagation and fragmentation. On the one hand, the ability of the framework to model the crack propagation under different dynamic loadings (impact and blast) is demonstrated by comparison with numerical and experimental results reported in the literature for elastic and elasto-plastic materials. On the other hand, the fragmentation due to the expansion of two different linear brittle specimens are studied to prove the ability of the framework to model multiple crack initiations. Once again the results are in good agreement with the ones reported in the literature. Therefore the DG/ECL framework is found to be a versatile tools, which can be applied for both dynamic crack propagation and fragmentation.

Acknowledgements Authors acknowledge the FNRS for its funding support to the researches and to the participation of CFRAC2011. Computational resources have been provided by the supercomputing facilities of the Consortium des Équipements de Calcul Intensif en Fédération Wallonie Bruxelles (CÉCI) funded by the Fond de la Recherche Scientifique de Belgique (FRS-FNRS).

\section{References}

Barsoum I, Faleskog J (2007) Rupture mechanisms in combined tension and shearmicromechanics. International Journal of Solids and Structures 44(17):5481 - 5498, DOI 10.1016/j.ijsolstr.2007.01.010

Becker G, Noels L (2011) A fracture framework for eulerbernoulli beams based on a full discontinuous galerkin formulation/extrinsic cohesive law combination. International Journal for Numerical Methods in Engineering 85(10):1227-1251, DOI 10.1002/nme.3008, URL http://dx.doi.org/10.1002/nme.3008

Becker G, Noels L (2012) A full discontinuous galerkin formulation of non-linear kirchhoff-love shells: elastoplastic finite deformations, parallel computation \& fracture applications. International Journal for Numerical Methods in Engineering Accepted:-

Becker G, Geuzaine C, Noels L (2011) A one field full discontinuous galerkin method for kirchhoff-love shells 
applied to fracture mechanics. Computer Methods in Applied Mechanics and Engineering 200(45-46):3223 3241, DOI 10.1016/j.cma.2011.07.008

Camacho GT, Ortiz M (1996) Computational modelling of impact damage in brittle materials. International Journal of Solids and Structures 33(20-22):2899-2938, URL http://www.sciencedirect.com/science/article/B6VJS3VTSPVJ-3/2/98fe3c31d0cee8e029d78d1631c6bbdd

Cirak F, Ortiz M (2001) Fully c1-conforming subdivision elements for finite deformation thin-shell analysis. International Journal for Numerical Methods in Engineering 51(7):813-833, DOI 10.1002/nme.182, URL http://dx.doi.org/10.1002/nme.182

Cirak F, Ortiz M, Schrder P (2000) Subdivision surfaces: a new paradigm for thin-shell finite-element analysis. International Journal for Numerical Methods in Engineering 47(12):2039-2072, DOI 10.1002/(SICI)10970207(20000430)47:12;2039::AID-NME872;3.0.CO;2-1

Cirak F, Ortiz M, Pandolfi A (2005) A cohesive approach to thin-shell fracture and fragmentation. Computer Methods in Applied Mechanics and Engineering 194(21-24):2604-2618, URL http://www.sciencedirect.com/science/article/B6V294FBW600-4/2/06c015e841d7fba329790dd872c4986e

Corigliano A, Cacchione F, Frangi A, Zerbini S (2008) Numerical modelling of impact rupture in polysilicon microsystems. Computational Mechanics 42:251259, URL http://dx.doi.org/10.1007/s00466-007-0231-5, 10.1007/s00466-007-0231-5

Cuitino A, Ortiz M (1992) A material-independent method for extending stress update algorithms from small-strain plasticity to finite plasticity with multiplicative kinematics. Engineering Computations 9:437-451

Deiterding R, Radovitzky R, Mauch S, Noels L, Cummings J, Meiron D (2006) A virtual test facility for the efficient simulation of solid material response under strong shock and detonation wave loading. Engineering with Computers 22(3):325-347-, URL http://dx.doi.org/10.1007/s00366-006-0043-9

Dickens SH, Cho BH (2005) Interpretation of bond failure through conversion and residual solvent measurements and weibull analyses of flexural and microtensile bond strengths of bonding agents. Dental Materials 21(4):354 - 364, DOI 10.1016/j.dental.2004.05.007

Dung NT, Wells GN (2008) Geometrically nonlinear formulation for thin shells without rotation degrees of freedom. Computer Methods in Applied Mechanics and Engineering 197(3340):2778 - 2788, DOI 10.1016/j.cma.2008.01.001

Engel G, Garikipati K, Hughes TJR, Larson MG, Mazzei L, Taylor RL (2002) Continuous/discontinuous finite element approximations of fourth-order elliptic problems in structural and continuum mechanics with applications to thin beams and plates, and strain gradient elasticity. Computer Methods in Applied Mechanics and Engineering 191(34):3669-3750, URL http://www.sciencedirect.com/science/article/B6V2945SRHD8-1/2/464ae949db39e8ad8352b23195051109

Gao X, Kim J (2006) Modeling of ductile fracture: Significance of void coalescence. International Journal of Solids and Structures 43(20):6277 - 6293, DOI 10.1016/j.ijsolstr.2005.08.008

Hansbo P, Larson MG (2002) A discontinuous galerkin method for the plate equation. Calcolo 39(1):41-59, URL http://dx.doi.org/10.1007/s100920200001

Hillerborg A, Modéer M, Petersson PE (1976) Analysis of crack formation and crack growth in concrete by means of fracture mechanics and finite elements. Cement and Concrete Research 6(6):773 - 781, DOI 10.1016/00088846(76)90007-7

Hulbert GM, Chung J (1996) Explicit time integration algorithms for structural dynamics with optimal numerical dissipation. Computer Methods in Applied Mechanics and Engineering 137(2):175 - 188, DOI DOI: 10.1016/S0045-7825(96)01036-5, URL http://www.sciencedirect.com/science/article/B6V293WFNRDY-5/2/2ec79b924e3dd1b9231ffe40c6a40306

Larsson R, Mediavilla J, Fagerström M (2011) Dynamic fracture modeling in shell structures based on xfem. Int $\mathrm{J}$ Numer Meth Engng 86(4-5):499-527, DOI 10.1002/nme.3086, URL http://dx.doi.org/10.1002/nme.3086

Leterrier Y, Andersons J, Pitton Y, Manson JAE (1997) Adhesion of silicon oxide layers on poly(ethylene terephthalate). ii: Effect of coating thickness on adhesive and cohesive strengths. Journal of Polymer Science Part B: Polymer Physics 35(9):1463-1472, DOI 10.1002/(SICI)10990488(19970715)35:9;1463::AID-POLB16;3.0.CO;2-4

Levy S (2010) Exploring the physics behind dynamic fragmentation through parallel simulations. $\mathrm{PhD}$ thesis, Ecole Polytechnique Fédérale de Lausanne, Lausanne, DOI 10.5075/epfl-thesis4898, URL http://library.epfl.ch/theses/?nr=4898, http://library.epfl.ch/theses/?nr=4898

Levy S, Molinari J, Radovitzky R (2012) Dynamic fragmentation of a brittle plate under biaxial loading: strength or toughness controlled? International Journal of Fracture 174:203-215, DOI 10.1007/s10704-012-9691-5, URL http://dx.doi.org/10.1007/s10704-012-9691-5

Li H, Chandra N (2003) Analysis of crack growth and crack-tip plasticity in ductile materials using cohesive zone models. International Journal of Plasticity 19(6):849-882-, URL http://www.sciencedirect.com/science/article/B6TWX45DF7DC-2/2/5b0f96fbff717dba3403fe5e02295e46 
Mergheim J, Kuhl E, Steinmann P (2004) A hybrid discontinuous galerkin/interface method for the computational modelling of failure. Communications in $\mathrm{Nu}-$ merical Methods in Engineering 20(7):511-519, URL http://dx.doi.org/10.1002/cnm.689

Molinari JF, Gazonas G, Raghupathy R, Rusinek A, Zhou F (2007) The cohesive element approach to dynamic fragmentation: the question of energy convergence. International Journal for Numerical Methods in Engineering 69(3):484-503, URL http://dx.doi.org/10.1002/nme.1777

Mota A, Knap J, Ortiz M (2008) Fracture and fragmentation of simplicial finite element meshes using graphs. International Journal for Numerical Methods in Engineering 73(11):1547-1570, URL http://dx.doi.org/10.1002/nme.2135

Nahshon K, Hutchinson J (2008) Modification of the gurson model for shear failure. European Journal of Mechanics - A/Solids 27(1):1 - 17, DOI 10.1016/j.euromechsol.2007.08.002

Needleman A (1987) A continuum model for void nucleation by inclusion debonding. Journal of Applied Mechanics 54:525-531

Needleman A (1990a) An analysis of decohesion along an imperfect interface. International Journal of Fracture 42:21-40, URL http://dx.doi.org/10.1007/BF00018611, 10.1007/BF00018611

Needleman A (1990b) An analysis of tensile decohesion along an interface. Journal of the Mechanics and Physics of Solids 38(3):289 - 324, DOI 10.1016/00225096(90)90001-K

Nielsen K, Hutchinson J (2011) Cohesive tractionseparation laws for tearing of ductile metal plates. International Journal of Impact Engineering DOI 10.1016/j.ijimpeng.2011.02.009

Nielsen KL, Tvergaard V (2009) Effect of a shear modified gurson model on damage development in a fsw tensile specimen. International Journal of Solids and Structures 46(34):587 - 601, DOI 10.1016/j.ijsolstr.2008.09.011

Noels L (2009) A discontinuous galerkin formulation of non-linear kirchhoff-love shells. International Journal for Numerical Methods in Engineering 78(3):296-323, URL http://dx.doi.org/10.1002/nme.2489

Noels L, Radovitzky R (2008) A new discontinuous galerkin method for kirchhoff-love shells. Computer Methods in Applied Mechanics and Engineering 197(33-40):2901-2929, URL http://www.sciencedirect.com/science/article/B6V294RSRDCT-1/2/9b24e4b7bb547bf5c27d28e6b0898610

Ortiz M, , Pandolfi A (2000) Finite-deformation irreversible cohesive elements for three-dimensional crack propagation analysis. International Journal for Numerical Methods in Engineering 44:44-1267
Pandolfi A, Ortiz M (2002) An efficient adaptive procedure for three-dimensional fragmentation simulations. Engineering with Computers 18(2):148-159, URL http://dx.doi.org/10.1007/s003660200013

Pandolfi A, Guduru P, Ortiz M, Rosakis A (2000) Three dimensional cohesive-element analysis and experiments of dynamic fracture in c300 steel. International Journal of Solids and Structures 37(27):3733 - 3760, DOI 10.1016/S0020-7683(99)00155-9

Papoulia KD, Sam CH, Vavasis SA (2003) Time continuity in cohesive finite element modeling. International Journal for Numerical Methods in Engineering 58(5):679-701, URL http://dx.doi.org/10.1002/nme.778

Papoulia KD, Vavasis SA, Ganguly P (2006) Spatial convergence of crack nucleation using a cohesive finite-element model on a pinwheel-based mesh. International Journal for Numerical Methods in Engineering 67(1):1-16, DOI 10.1002/nme.1598, URL http://dx.doi.org/10.1002/nme.1598

Prechtel M, Leugering G, Steinmann P, Stingl M (2011) Towards optimization of crack resistance of composite materials by adjustment of fiber shapes. Engineering Fracture Mechanics 78(6):944 - 960, DOI 10.1016/j.engfracmech.2011.01.007

Radovitzky R, Seagraves A, Tupek M, Noels L (2011) A scalable $3 \mathrm{~d}$ fracture and fragmentation algorithm based on a hybrid, discontinuous galerkin, cohesive element method. Computer Methods in Applied Mechanics and Engineering 200(1-4):326 - 344, DOI 10.1016/j.cma.2010.08.014

Scheider I, Brocks W (2003) Simulation of cupcone fracture using the cohesive model. Engineering Fracture Mechanics 70(14):1943-1961, URL http://www.sciencedirect.com/science/article/B6V2R48KF7Y8-1/2/3ebd02d524ce26db2ca47735385023d9

Scheider I, Brocks W (2006) Cohesive elements for thin-walled structures. Computational Materials Science 37(12):101 - 109, DOI 10.1016/j.commatsci.2005.12.042, ¡ce:title ¿Proceedings of the 14th International Workshop on Computational Mechanics of Materials;/ce:title $i$; xocs:full-name $_{i}$ 14th International Workshop on Computational Mechanics of Materials;/xocs:full-name;

Scheyvaerts F, Onck P, Tekoglu C, Pardoen T (2011) The growth and coalescence of ellipsoidal voids in plane strain under combined shear and tension. Journal of the Mechanics and Physics of Solids 59(2):373 - 397, DOI 10.1016/j.jmps.2010.10.003

Simo J, Kennedy J (1992) On a stress resultant geometrically exact shell model. part $\mathrm{v}$. nonlinear plasticity: formulation and integration algorithms. Computer Methods in Applied Mechanics and Engineering 96(2):133 171, DOI DOI: 10.1016/0045-7825(92)90129-8, URL 
http://www.sciencedirect.com/science/article/B6V2947YJHG3-T/2/9de358cec2b4c5890934d756457471dc

Simo J, Fox D, Rifai M (1990a) On a stress resultant geometrically exact shell model. part iii: Computational aspects of the nonlinear theory. Computer Methods in Applied Mechanics and Engineering 79(1):21 70, DOI DOI: 10.1016/0045-7825(90)90094-3, URL http://www.sciencedirect.com/science/article/B6V2947XSWMP-3/2/a7d8adf2ee1bde1ca1943b2179e27a9a

Simo JC, Fox DD (1989) On stress resultant geometrically exact shell model. part i: formulation and optimal parametrization. Comput Methods Appl Mech Eng 72(3):267-304

Simo JC, Fox DD, Rifai MS (1989) On a stress resultant geometrically exact shell model. part ii: the linear theory; computational aspects. Comput Methods Appl Mech Eng 73(1):53-92

Simo JC, Rifai MS, Fox DD (1990b) On a stress resultant geometrically exact shell model. part iv: Variable thickness shells with through-thethickness stretching. Computer Methods in Applied Mechanics and Engineering 81(1):91 - 126, DOI DOI: 10.1016/0045-7825(90)90143-A, URL http://www.sciencedirect.com/science/article/B6V294808WRV-30/2/57f79632994b5829488baed916c1a998

Süli E, Mozolevski I (2007) hp-version interior penalty dgfems for the biharmonic equation. Computer Methods in Applied Mechanics and Engineering 196(1316):1851 1863, DOI 10.1016/j.cma.2006.06.014

Tvergaard V (1990) Effect of fibre debonding in a whiskerreinforced metal. Materials Science and Engineering: A 125(2):203 - 213, DOI 10.1016/0921-5093(90)90170-8

Tvergaard V, Hutchinson JW (1992) The relation between crack growth resistance and fracture process parameters in elastic-plastic solids. Journal of the Mechanics and Physics of Solids 40(6): 1377 - 1397, DOI 10.1016/00225096(92)90020-3

Tvergaard V, Hutchinson JW (1993) The influence of plasticity on mixed mode interface toughness. Journal of the Mechanics and Physics of Solids 41(6):1119 - 1135, DOI 10.1016/0022-5096(93)90057-M

Tvergaard V, Hutchinson JW (1996) Effect of straindependent cohesive zone model on predictions of crack growth resistance. International Journal of Solids and Structures 33(20-22):3297-3308, URL http://www.sciencedirect.com/science/article/B6VJS3VTSPVJ-S/2/71976b9761cf9bd81d308e234b91cf3b

Weibull W (1939) A statistical theory of strength of materials. vol Proceedings of the Ingeniors Vetenskapsakad, $p$ 151

Wells GN, Dung NT (2007) A c0 discontinuous galerkin formulation for kirchhoff plates. Computer Methods in Applied Mechanics and Engineering 196(35-36):3370-3380
Wyart E, Coulon D, Duflot M, Pardoen T, Remacle JF, Lani F (2007) A substructured fe-shell/xfe-3d method for crack analysis in thin-walled structures. International Journal for Numerical Methods in Engineering 72(7):757-779, DOI 10.1002/nme.2029

$\mathrm{Xu}$ XP, Needleman A (1994) Numerical simulations of fast crack growth in brittle solids. Journal of the Mechanics and Physics of Solids 42(9):1397 - 1434, DOI 10.1016/0022-5096(94)90003-5

Zavattieri P (2004) Modeling of crack propagation in thinwalled structures. Mecanica Computacional XXIII:209228

Zavattieri PD (2006) Modeling of crack propagation in thin-walled structures using a cohesive model for shell elements. J Appl Mech 73(6):948-958, URL http://link.aip.org/link/?AMJ/73/948/1

Zhang ZJ, Paulino GH, Celes W (2007) Extrinsic cohesive modelling of dynamic fracture and microbranching instability in brittle materials. International Journal for Numerical Methods in Engineering 72(8):893-923, URL http://dx.doi.org/10.1002/nme.2030

Zhou F, Molinari JF (2004a) Dynamic crack propagation with cohesive elements: a methodology to address mesh dependency. International Journal for Numerical Methods in Engineering 59(1):1-24, DOI 10.1002/nme.857, URL http://dx.doi.org/10.1002/nme.857

Zhou F, Molinari JFc (2004b) Stochastic fracture of ceramics under dynamic tensile loading. International Journal of Solids and Structures 41(22-23):6573 - 6596, DOI DOI: 10.1016/j.ijsolstr.2004.05.029 